\title{
DINOFLAGELADOS DEL EOCENO MEDIO A SUPERIOR DE LAS CUENCAS PUNTA DEL ESTE Y COLORADO, LATITUDES MEDIAS DEL ATLÁNTICO SUDOCCIDENTAL
}

\author{
GLORIA DANERS \\ Departamento de Paleontología, Instituto de Ciencias Geológicas, Facultad de Ciencias, Universidad de la República, \\ Iguá 4225, CP 11400, Montevideo, Uruguay.glo@fcien.edu.uy \\ G. RAQUEL GUERSTEIN \\ Departamento de Geología, Instituto Geológico del Sur, Universidad Nacional del Sur, CONICET, San Juan 670, \\ B8000ICN, Bahía Blanca, Argentina. raquel.guerstein@uns.edu.ar
}

CECILIA R. AMENÁBAR

Instituto Antártico Argentino, Instituto de Estudios Andinos Don Pablo Groeber-CONICET, Departamento de Ciencias Geológicas, Universidad de Buenos Aires, Intendente Guiraldes 2160, C1428EGA, Buenos Aires, Argentina. amenabar@gl.fcen.uba.ar

ETHEL MORALES

Departamento de Geología Sedimentaria y Aplicada, Instituto de Ciencias Geológicas, Facultad de Ciencias, Universidad de la República, Iguá 4225, CP 11400, Montevideo, Uruguay. ethel@fcien.edu.uy

\begin{abstract}
MIDDLE TO UPPER EOCENE DINOFLAGELLATE CYSTS FROM PUNTA DEL ESTE AND COLORADO BASINS, MID LATITUDE SOUTHWEST ATLANTIC OCEAN. The analysis of middle Eocene dinoflagellate cyst assemblages from sites at Punta del Este $\left(\sim 36^{\circ} \mathrm{S}\right.$, Uruguay) and Colorado ( $38^{\circ} \mathrm{S}$, Argentina) basins allows to interpret ocean circulation patterns on the South American Shelf prior to the opening of the Drake Passage. The assemblages contain endemic-Antarctic taxa such as Enneadocysta, Deflandrea, Vozzhennikovia and Spinidinium, which are also dominant in several circum-Antarctic sites, located southern $45^{\circ} \mathrm{S}$. Enneadocysta dictyostila is the most abundant species recorded from the studied sites and the unique member of the endemic assemblage apparently tolerant to warm surface waters. The presence of endemic taxa at mid latitudes has been related to a strong clockwise subpolar gyre favoured by the partial continental blockage of the Tasmanian Gateways and the Drake Passage. The distribution of the dinoflagellate cyst assemblages along the South Atlantic Ocean Shelf can be explained by a similar dynamical mechanism induced by a cyclonic subpolar gyre on the South Atlantic Ocean. The western boundary current of this gyre, starting on the west Antarctic continental slope, would follow a similar path to the present Malvinas Current on the Patagonian slope, which introduce cold oceanic waters to the shelf and intensifies the northward shelf transport. In the same way, during the middle Eocene the western boundary current of a proto-Weddell Gyre transported the circum-Antarctic waters and the endemic taxa northward along the Southwestern Atlantic Shelf. The opening and deepening of the Tasmanian Gateway and Drake Passage and the development of an incipient Antarctic Circumpolar Current during the latest Eocene-Oligocene disrupted the subpolar gyres and promoted the extinction of the endemic species.
\end{abstract}

Key words: dinoflagellate cysts, Eocene, Punta del Este Basin, Colorado Basin, biostratigraphy, paleoceanography.

RESUMO - O estudo de associações de cistos de dinoflagelados do Eoceno médio nas bacias de Punta del Este $\left(\sim 36^{\circ} \mathrm{S}\right.$, Uruguai) e do Colorado ( $\sim 38^{\circ} \mathrm{S}$, Argentina) permite interpretar os padrões de circulação oceânica na plataforma sul-americana antes da abertura da Passagem de Drake. As associações são caracterizadas pela presença de táxons antárticos endémicos, como Enneadocysta, Deflandrea, Vozzhennikovia e Spinidinium, dominantes em vários locais da região Circumpolar Antárctica localizados ao sul do $45^{\circ} \mathrm{S}$. A espécie mais abundante registrada nos locais estudados é Enneadocysta dictyostila, que é o único membro da associação endêmica aparentemente resistente às águas aquecidas na superfície. Pesquisas anteriores já haviam mencionado a presença de táxons endêmicos em latitudes médias a um forte giro subpolar no sentido horário favorecido pelo bloqueio parcial das passagens da Tasmânia e de Drake. Propõe-se aqui, que a distribuição das associações de cistos de dinoflagelados, ao longo das bacias da margem continental do Atlântico Sul, pode ser explicada por um mecanismo dinâmico semelhante induzido por um giro subpolar ciclônico no Oceano Atlântico Sul. O atual limite ocidental deste giro, começando no talude continental da Antártica ocidental, iria seguir um caminho semelhante ao presente atual Corrente das Malvinas na encosta da Patagônia, que introduz as águas oceânicas frias na plataforma e intensifica o transporte das águas na plataforma para o norte. De maneira similar, durante o Mesoeoceno, o limite ocidental da corrente Protogiro de 
Weddell transportou as águas circum-antárticas e os táxons endêmicos para o norte ao longo da margem Atlântico Sudoeste. A abertura e o aprofundamento das passagens de Tasmânia e Drake, assim como o desenvolvimento de uma incipiente corrente circumpolar Antártica no final do Eoceno e início do Oligoceno, interromperam os giros subpolares e promoveram a extinção das espécies endêmicas.

Palavras-chave: cistos de dinoflagelados, Eoceno, Bacia de Punta del Este, Bacia Colorado, bioestratigrafia, paleoceanografía.

\section{INTRODUCCIÓN}

Los climas del Paleógeno temprano fueron considerablemente más cálidos que los actuales, con concentraciones de $\mathrm{CO}_{2}$ atmosférico más altas y gradientes térmicos latitudinales significativamente menores (Greenwood \& Wing, 1995; Sexton et al., 2006; Sluijs et al., 2006; Zachos et al., 2008; Bijl et al., 2009). La transición de un clima cálido en el Eoceno temprano a uno frío en el Oligoceno se inició hace aproximadamente $50 \mathrm{Ma}$, cuando comenzó el enfriamiento de las aguas del proto-Océano Austral (Zachos et al., 2001, 2008; Bijl et al., 2009, 2013b). Esta transición fue brevemente interrumpida por eventos hipertermales, entre ellos el Óptimo Climático del Eoceno medio (Bohaty \& Zachos, 2003; Zachos et al., 2008; Bohaty et al., 2009; Bijl et al., 2010). El enfriamiento global se aceleró durante el Eoceno medio a tardío, provocando el establecimiento de grandes mantos de hielo en Antártida hacia fines del Eoceno (Zachos et al., 1994; Barker et al., 2007). Los cambios tectónicos ocurridos durante este período en el Hemisferio Sur, tales como la apertura y profundización del Pasaje de Drake y el Conducto de Tasmania, generaron en primer lugar la formación de aguas intermedias relativamente frías (Bijl et al., 2013a) y subsecuentemente el establecimiento de una corriente circumpolar irrestricta (Stickley et al., 2004a; Scher \& Martin, 2006; Livermore et al., 2007; Lagabrielle et al., 2009).

Los quistes de dinoflagelados de pared orgánica son un grupo de palinomorfos, en su mayoría de origen marino, utilizados con excelentes resultados para analizar la transición invernadero-glaciar durante el Paleógeno (Sluijs et al., 2005). Son producidos por organismos eucariontes que constituyen un componente importante del plancton marino y mucho de estos quistes de resistencia son recuperados de manera abundante en el registro fósil. Las asociaciones de quistes de dinoflagelados son muy importantes en los estudios del Paleógeno, ya que incluyen numerosas especies con significado bioestratigráfico y permiten estimar las condiciones físico-químicas de las aguas superficiales en el pasado (Sluijs et al., 2005; Bijl et al., 2011, 2013a,b; Guerstein et al., 2014; González Estebenet, 2014b). Los quistes de dinoflagelados se preservan de manera preferencial en ambientes con altas tasas de sedimentación y bajas concentraciones de oxígeno, como en sedimentos de las plataformas continentales desarrolladas durante el Paleógeno en áreas circum-antárticas y en el Atlántico Sudoccidental (Wrenn \& Hart, 1988; Brinkhuis et al., 2003a,b; Guerstein et al., 2010; Bijl et al., 2011).

Las asociaciones de quistes de dinoflagelados experimentaron cambios muy significativos como respuesta a la variación de los patrones de circulación oceánica que generaron las modificaciones tectónicas antes mencionadas.
Las asociaciones del Paleoceno y Eoceno temprano están dominadas por especies cosmopolitas y hace $\sim 50$ Ma revelan un marcado aumento en los porcentajes de especies endémicas de altas latitudes (Bijl et al., 2011, 2013b). Esta asociación, que denominamos asociación endémica-antártica, fue originalmente registrada como "Flora Trasantártica" por Wrenn \& Beckman (1982). Estudios posteriores reconocieron a las especies pertenecientes a esta comunidad antártica en numerosas localidades del Océano Austral (Wrenn \& Hart, 1988; Cocozza \& Clarke, 1992; Mao \& Mohr, 1995; Truswell, 1997; Levy \& Hardwood, 2000; Brinkhuis et al., 2003a,b; MacPhail \& Truswell, 2004; Guerstein et al., 2008b; Sluijs et al., 2009; Bijl et al., 2011, 2013a,b; Guerstein et al., 2014; González Estebenet et al., 2014a,b). La asociación endémica-antártica proliferó hasta el final del Eoceno, cuando se modificaron los patrones de circulación oceánica como consecuencia de la profundización del Pasaje de Drake y el Conducto de Tasmania (Sluijs et al., 2003; Stickley et al., 2004a; Guerstein et al., 2008b; Houben et al., 2011, 2013). Dichos cambios provocaron la consecuente extinción de las especies endémicas, que fueron reemplazadas por taxa cosmopolitas (Stickley et al., 2004b; Guerstein et al., 2010; Bijl et al., 2011; Houben et al., 2013; González Estebenet et al., 2015).

Este grupo de microfósiles muestra un alto potencial como indicador indirecto (proxy data) para comparar los resultados de modelos de circulación oceánica puestos a prueba sobre registros de latitudes altas del Hemisferio Sur (Huber et al., 2004; Bijl et al., 2013a; González Estebenet et al., 2014a). Hasta el momento son escasos y de poca potencia los intervalos estratigráficos asignados al Eoceno en localidades de latitudes medias del Atlántico Sudoccidental (Guerstein et al., 2010). Sin embargo, son muy importantes los registros provenientes de datos de subsuelo de las cuencas Punta del Este (Uruguay) y Colorado (Argentina), los cuales revelan la presencia de algunos de los miembros de la asociación endémica (Guerstein \& Daners, 2010; Guerstein et al., 2008a, 2015). Estudios previos desarrollados en el área de Tasmania relacionaron la presencia de especies endémicas en latitudes medias con un giro subpolar en sentido ciclónico favorecido por la obstrucción parcial de los pasajes de Tasmania y Drake (Huber et al., 2004; Stickley et al., 2004a; Bijl et al., 2011). La distribución de quistes de dinoflagelados en la Plataforma del Océano Atlántico Sudoeste puede ser explicada por un mecanismo dinámico similar, inducido por un giro subpolar ciclónico en el Atlántico Sur (Guerstein \& Daners, 2010; González Estebenet et al., 2014a).

En este trabajo se analizan las asociaciones de quistes de dinoflagelados asignadas al Eoceno medio a superior del Pozo Gaviotín, realizado sobre la plataforma continental uruguaya en la Cuenca Punta del Este (Figura 1). Los 


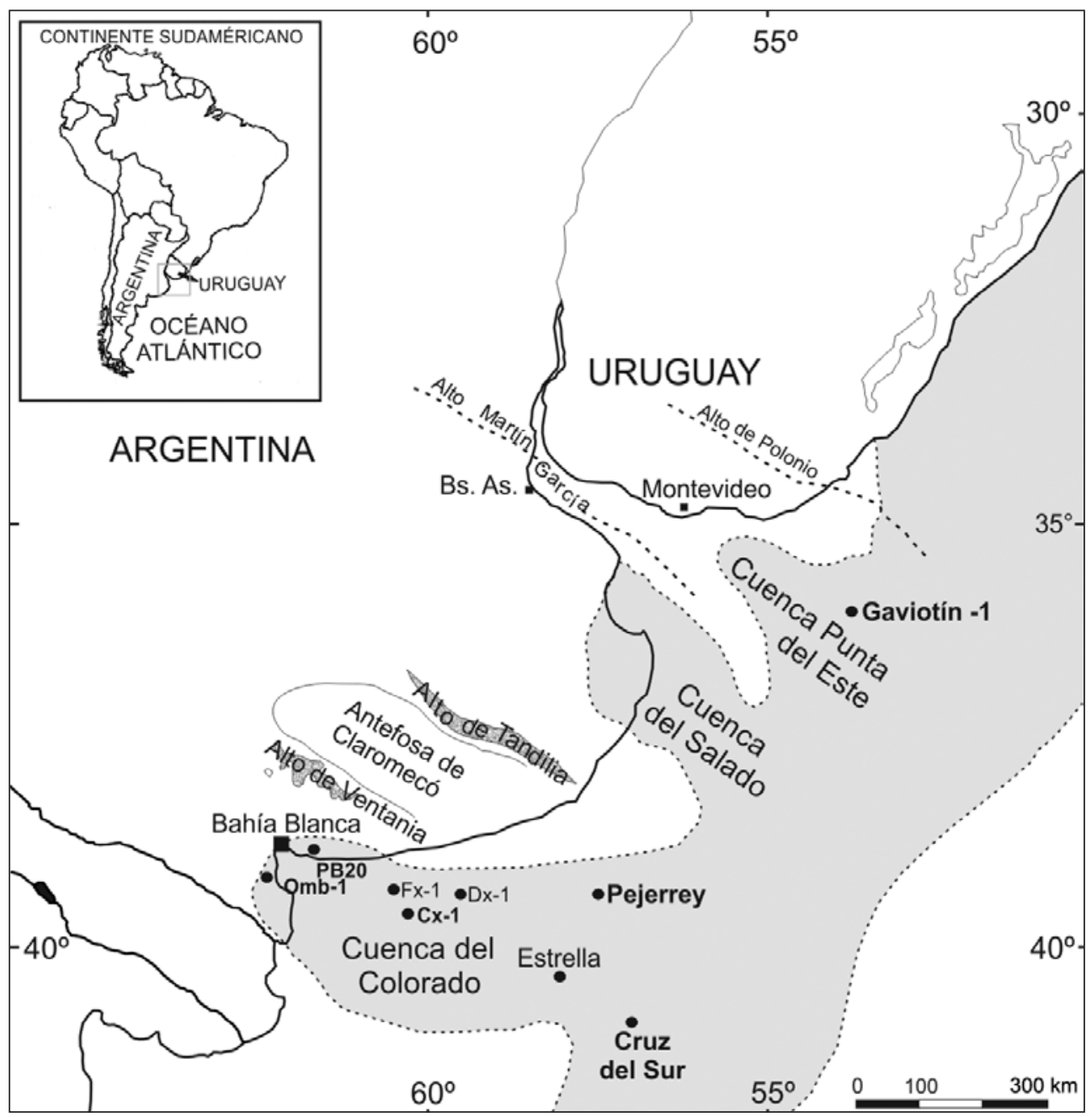

Figura 1. Mapa de ubicación de las cuencas Punta del Este y Colorado, mostrando los sitios analizados (modificado de Urien \& Zambrano, 1996).

Figure 1. Location map of the Punta del Este and Colorado basins showing the compiled sites (modified from Urien \& Zambrano, 1996).

resultados se comparan con las asociaciones coetáneas de la Cuenca del Colorado, dando lugar a un análisis conjunto de seis perforaciones offshore y dos onshore localizadas entre los $36^{\circ} \mathrm{S} \mathrm{y} 41^{\circ} \mathrm{S}$ con el objeto de interpretar los patrones de circulación oceánica superficial sobre la plataforma sudamericana en un escenario tectónico previo a la profundización del Pasaje de Drake.

\section{MARCO GEOLÓGICO}

La apertura del Atlántico Sur es el resultado de un complejo proceso de ruptura continental y magmatismo sobre los márgenes conjugados de la parte sur de África y Sudamérica que se propagó en forma diacrónica desde el sur hacia el norte (Uchupi \& Emery, 1991; Gladczenko et al., 1997; Heine et al., 2013). Se relaciona con la última fase de la segmentación de Gondwana y la ruptura inicial ocurrió aproximadamente a los $130 \mathrm{Ma}$ (M10) entre los $44^{\circ} \mathrm{S}$ y $45^{\circ}$ S (Moulin et al., 2005; Ghidella et al., 2006).

Los márgenes continentales argentino y uruguayo han sido definidos como típicos márgenes pasivos de rift volcánico, a través del análisis de los reflectores sísmicos profundos en la plataforma y talud continental (Hinz et al., 1999; Franke et al., 2006, 2007; Soto et al., 2011).

Las cuencas del Colorado y Salado, ubicadas en el margen argentino, y la Cuenca Punta del Este, desarrollada sobre la plataforma continental uruguaya, corresponden a una serie de aulacógenos, cuyos ejes están orientados en forma transversal a los márgenes continentales. La Cuenca Punta del Este se extiende hacia el norte hasta el Alto de Polonio que la separa de la Cuenca de Pelotas y limita al sur a través del Alto Martín García con la Cuenca del Salado. En tanto, esta última se extiende hacia el sur hasta el Alto de Tandilia que la separa de la Cuenca del Colorado (Figura 1).

Se reconocen cuatro etapas en la evolución de estas cuencas desde el punto de vista tectono-estratigráfico: prerift (Paleozoico), rift (Jurásico-Cretácico Inferior), transición (Barremiano-Aptiano) y margen pasivo o deriva (AptianoPresente) (Stoakes et al., 1991; Fryklund et al., 1996; Ucha et al., 2003; Morales, 2013; Conti, 2015).

El paquete sedimentario correspondiente a la fase de margen pasivo constituye el mayor espesor sedimentario para 
el margen argentino-uruguayo (Franke et al., 2007), siendo el resultado de la interacción entre la subsidencia termal y flexural de las cuencas, el abastecimiento sedimentario y los cambios en el nivel de base (Morales, 2013; Conti, 2015).

Las pelitas que contienen el límite Cretácico/Paleógeno constituyen la primera transgresión marina que alcanza la posición de los pozos offshore de las cuencas Colorado, Salado y Punta del Este (Tavella \& Wrigth, 1996; Morales, 2013; Conti, 2015). Durante el Cenozoico la Patagonia extrandina se vio afectada por sucesivas transgresiones atlánticas de carácter regional (Malumián \& Nañez, 2011). En particular, en el Eoceno medio a tardío tuvo lugar un ciclo transgresivoregresivo que inundó gran parte de la Cuenca Austral. Este evento también ha sido reconocido en las cuencas del Punta del Este y Colorado, donde los registros correspondientes a este ciclo provienen exclusivamente de perforaciones profundas realizadas sobre la plataforma (Guerstein et al., 2010).

\section{MATERIAL Y MÉTODOS}

Para este trabajo se analizaron las asociaciones de quistes de dinoflagelados provenientes del intervalo entre los 1476 y $1576 \mathrm{~m}$ de profundidad del Pozo Gaviotín, realizado sobre la plataforma continental uruguaya en la Cuenca Punta del Este. Las muestras fueron procesadas en los laboratorios palinológicos del Departamento de Paleontología del Instituto de Ciencias Geológicas, Facultad de Ciencias de la Universidad de la República, Montevideo, Uruguay y de Petrobras, Río de Janeiro, Brasil. El tratamiento de extracción fisicoquímica consistió en la eliminación de la fracción inorgánica con ácidos clorhídrico y fluorhídrico. La fracción orgánica se concentró mediante filtrados con tamiz de $10 \mu \mathrm{m}$. Los residuos fueron montados en gelatinaglicerina. Los preparados están archivados en el repositorio de Paleopalinología del Departamento de Paleontología perteneciente al Instituto de Ciencias Geológicas de la Facultad de Ciencias, de la Universidad de la República, Montevideo, Uruguay.

A los efectos de comparar los resultados obtenidos en la Cuenca Punta del Este se re-estudiaron las asociaciones de quistes de dinoflageladas de la Cuenca del Colorado de intervalos estratigráficos previamente asignados al Eoceno de las perforaciones offshore Cx-1, Pejerrey-1 y Cruz del Sur y de los pozos Ombucta-1 y Puerto Belgrano 20, realizados en el sur de la Provincia de Buenos Aires (Figura 1). Las muestras de la Cuenca del Colorado se procesaron en los laboratorios del Servicio Geológico Canadiense, Dartmouth, Nova Scotia; en el Museo Argentino de Ciencias Naturales Bernardino Rivadavia, Buenos Aires, Argentina (MACN) y en el de INGEOSUR, Bahía Blanca. Una vez eliminada la fracción inorgánica con ácidos clorhídrico y fluorhídrico la fracción orgánica fue filtrada con tamices de 10, 25 y $180 \mu \mathrm{m}$. Los residuos de las muestras de las perforaciones Pejerrey-1 y Cx-1 fueron teñidos con Bismarck C; todos los residuos fueron montados en gelatina-glicerina con excepción de los de las muestras de la perforación $\mathrm{Cx}-1$ que fueron montados en celulosa Hidroxietil y pegados con elvacita. Los preparados están archivados en la colección palinológica de INGEOSUR. En la Tabla 1 se listan las muestras analizadas en metros de profundidad bajo boca de pozo, la colección donde se encuentran archivados los preparados palinológicos y la compañía que realizó cada perforación.

El estudio taxonómico se realizó bajo un microscopio óptico Olympus BX40 y una cámara digital Infinity1 del Instituto de Ciencias Geológicas, Facultad de Ciencias, Universidad de la República Montevideo, Uruguay, y un microscopio Nikon Eclipse 600 con una cámara digital Micrometrics del INGEOSUR, Departamento de Geología de la Universidad Nacional del Sur. Los ejemplares ilustrados en las Figuras 2-4 se identificaron con el número de muestra y las referencias England Finder (EF). En la Tabla 2 se listan las especies mencionadas en el texto de acuerdo a la base de datos DINOFLAJ2 de Fensome et al. (2008) y a los estudios taxonómicos realizados por Sluijs et al. (2009) y Williams et al. (2015).

Para el análisis bioestratigráfico se utilizaron los eventos de dinoflagelados calibrados en la plataforma de Tasmania con la Escala de Polaridad Geomagnética de Vandenberghe et al. (2012) usando magnetoestratigrafía y estratigrafía basada en isótopos estables (Bijl et al., 2013b). Debido a las características del muestreo (cuttings), para el análisis bioestratigráfico se consideraron principalmente los datos de últimas ocurrencias o techos estratigráficos y de manera complementaria se utilizaron en algunos casos los eventos de primeras ocurrencias o primeras ocurrencias abundantes.

Las asociaciones se caracterizaron de manera semicuantitativa utilizando la siguiente escala: muy raro (1), raro (hasta 5), frecuente (hasta 20), común (hasta 40) y abundante (más de 40) ejemplares por preparado (Tablas 3-4 y Figura 5). Se complementó el análisis de las asociaciones aquí estudiadas con registros de secciones coetáneas de la Cuenca del Colorado del pozo Estrella-1, calibradas con foraminíferos y nanofósiles y de las perforaciones Dx-1 y Fx-1 (Guler \& Guerstein, 2002).

\section{RESULTADOS}

\section{Cuenca Punta del Este}

Pozo Gaviotín. De las 41 muestras procesadas en este pozo para el análisis palinológico se estudiaron ocho muestras dentro de un intervalo de $100 \mathrm{~m}$ de espesor, entre 1476 y 1576 $\mathrm{m}$ bajo boca de pozo ( $\mathrm{mbp}$ ), parte del cual había sido asignado previamente por Daners \& Guerstein (2004) al Eoceno medio. Se reconocieron 31 especies de quistes de dinoflagelados, de las cuales 6 corresponden a la asociación endémica de altas latitudes del Hemisferio Sur (Asociación endémicaantártica) y las restantes tienen una distribución cosmopolita. En la Tabla 3 se muestran las frecuencias semicuantitativas resultantes del análisis de un preparado por nivel. Entre las especies endémicas, Enneadocysta dictyostila se presenta de manera abundante en todas las asociaciones, mientras que E. brevistila se encuentra bien representada, principalmente entre los 1446 y $1521 \mathrm{mbp}$. En esos niveles también se presenta de manera frecuente otra especie endémica, 

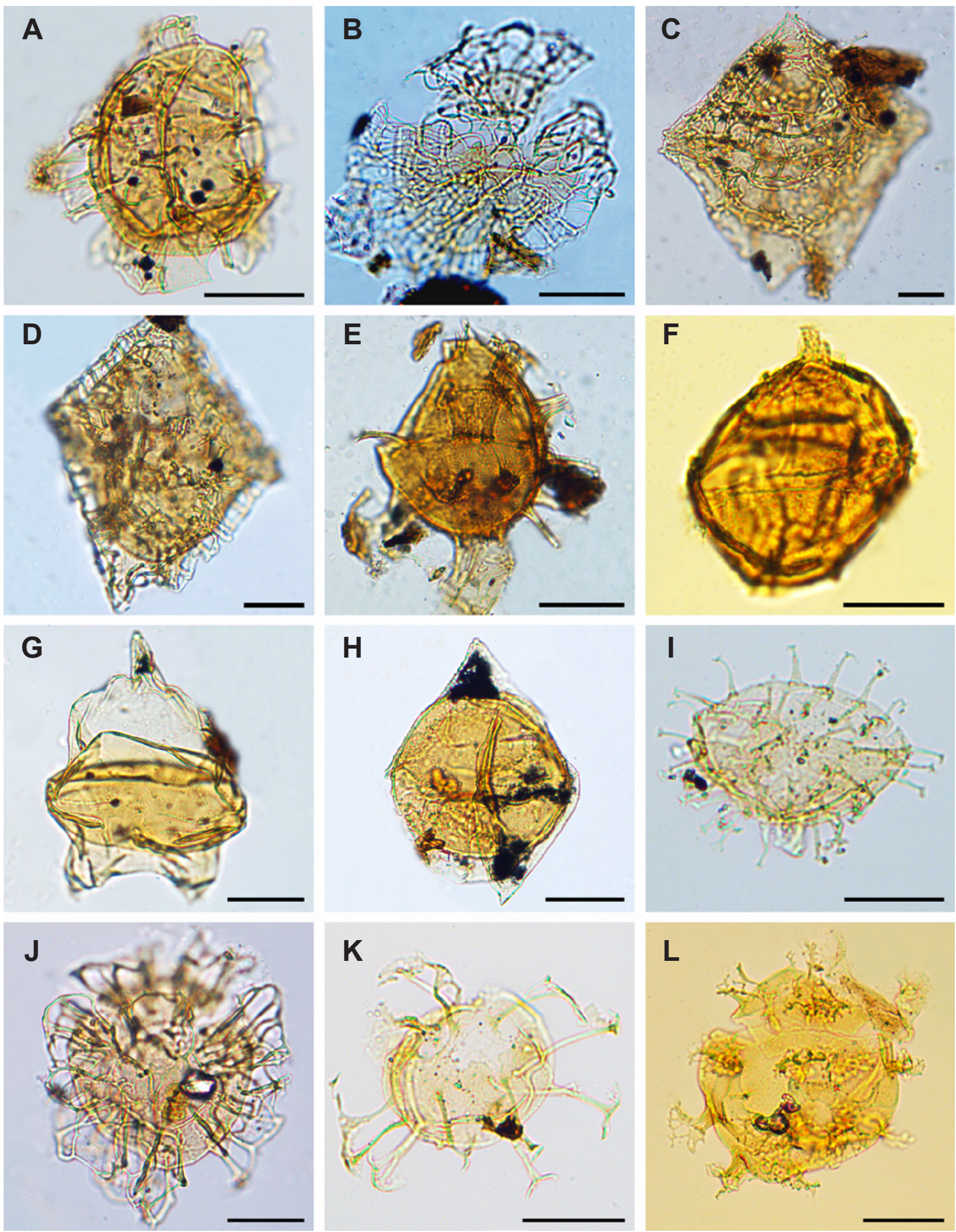

Figura 2/Figure 2. Quistes de dinoflagelados seleccionados del Pozo Gaviotín, Cuenca Punta del Este. Los ejemplares se identifican mediante el número de muestra y referencias del England Finder/Selected dinoflagellate cysts from the Gaviotín borehole, Punta del Este Basin. Specimens are identified by sample number and England Finder references. A, Achilleodinium biformoides (Eisenack, 1954) Eaton, 1976 (1549A, W26); B, Adnatosphaeridium multispinosum Williams \& Downie, 1966 (1576E0, H19/4); C, Charlesdowniea sp. 1 (1585C, V13); D, Charlesdowniea sp. 2 (1658A, S27/4); E, Cordosphaeridium sp. (1549F, S18/4-T18/2); F, Cribroperidinium giuseppei (Morgenroth, 1966) Helenes, 1984 (1549mB2, V22/1); G, Deflandrea antarctica Wilson, 1967 (1576A, J28); H, Deflandrea phosphoritica Eisenack, 1938 (1549B, G31); I, Diphyes colligerum (Deflandre \& Cookson, 1955) Cookson, 1965 emend. Goodman \& Witmer, 1985 (1476D, H15); J, Emmetrocysta urnaformis (Cookson, 1953) Stover, 1975 (1576A6, P29/3); K, Enneadocysta arcuata (Eaton, 1971) Stover \& Williams, 1995 (1476D, EFV8/2); L, Enneadocysta brevistila Fensome et al., 2006 (1512D, O30/2-O31/1). Escalas/ Scale bars $=20 \mu \mathrm{m}$. 

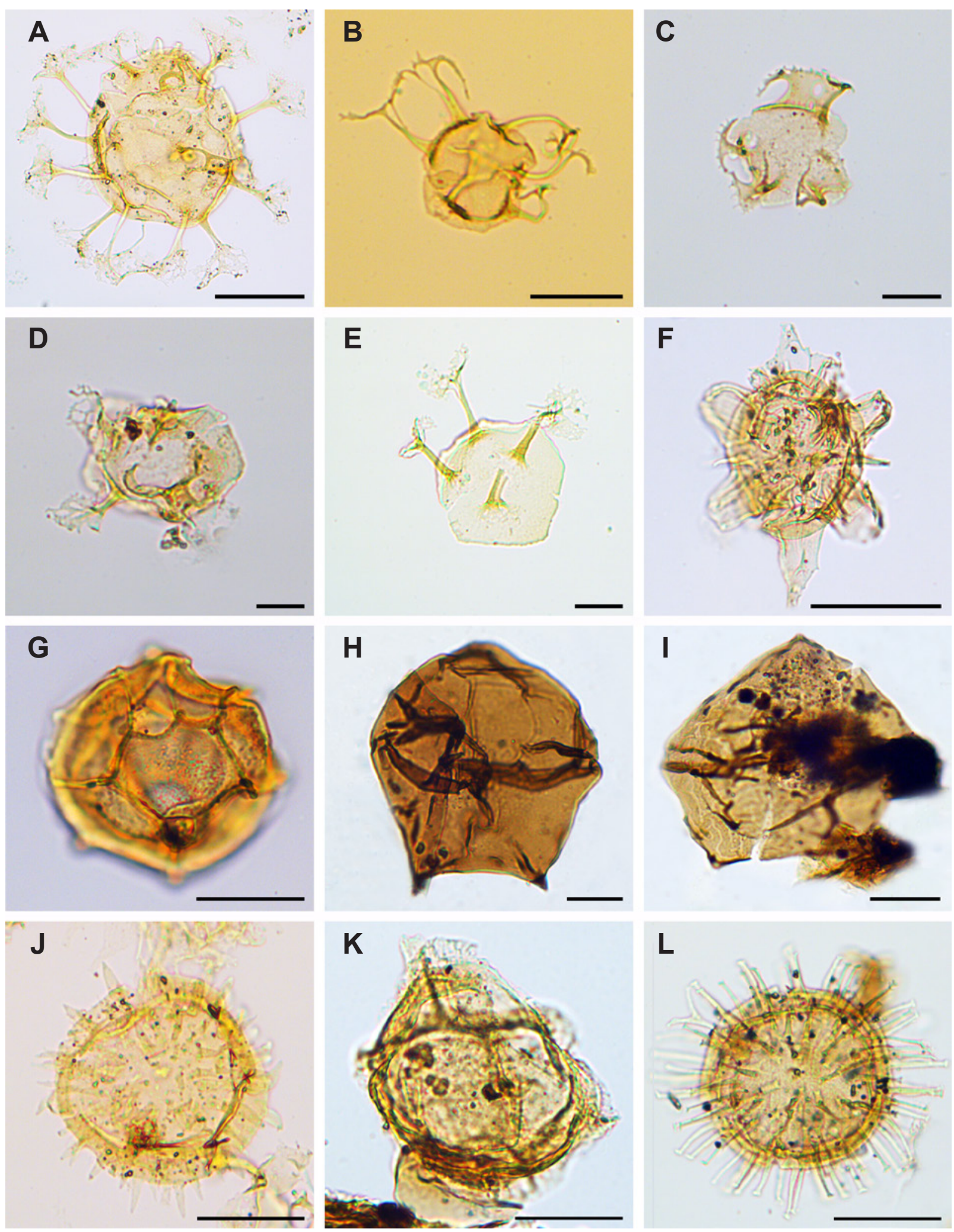

Figura 3/Figure 3. Quistes de dinoflagelados seleccionados del Pozo Gaviotín, Cuenca Punta del Este. Los ejemplares se identifican mediante el número de muestra y referencias del England Finder. / Selected dinoflagellate cysts from the Gaviotín borehole, Punta del Este Basin. Specimens are identified sample number and England Finder references. A, Enneadocysta dictyostila (Menéndez) Fensome et al., 2006 (1476A, U36); B, Enneadocysta arcuata, opérculo (1512D, N33/4); C, Enneadocysta sp. cf. E. arcuata, opérculo / operculum (1476D, K17/3-L17); D, Enneadocysta brevistila, opérculo / operculum (1512D, X13); E, Enneadocysta dictyostila, opérculo / operculum (1476D, M16/4); F, Hystrichokolpoma salacia Eaton, 1976 (1549A, V25/4); G, Impagidinium sp. (1476D, W11/3); H, Lejeunecysta sp. 1 (1558A, S25/4-S26/3); I, Lejeunecysta sp. 2 (1558C, F23/1); J, Lingulodinium machaerophorum (Deflandre \& Cookson, 1955) Wall, 1967 (1476A, E33/1-E33/2); K, Muratodinium fimbriatum (Cookson \& Eisenack, 1967) Drugg, 1970 (1549B, J22/4); L, Operculodinium centrocarpum (Deflandre \& Cookson) Wall, 1967 (1549C, H30/3-J30/1). Escalas/ Scale bars: A, F-L = $20 \mu \mathrm{m} ; \mathrm{B}-\mathrm{E}=10 \mu \mathrm{m}$. 

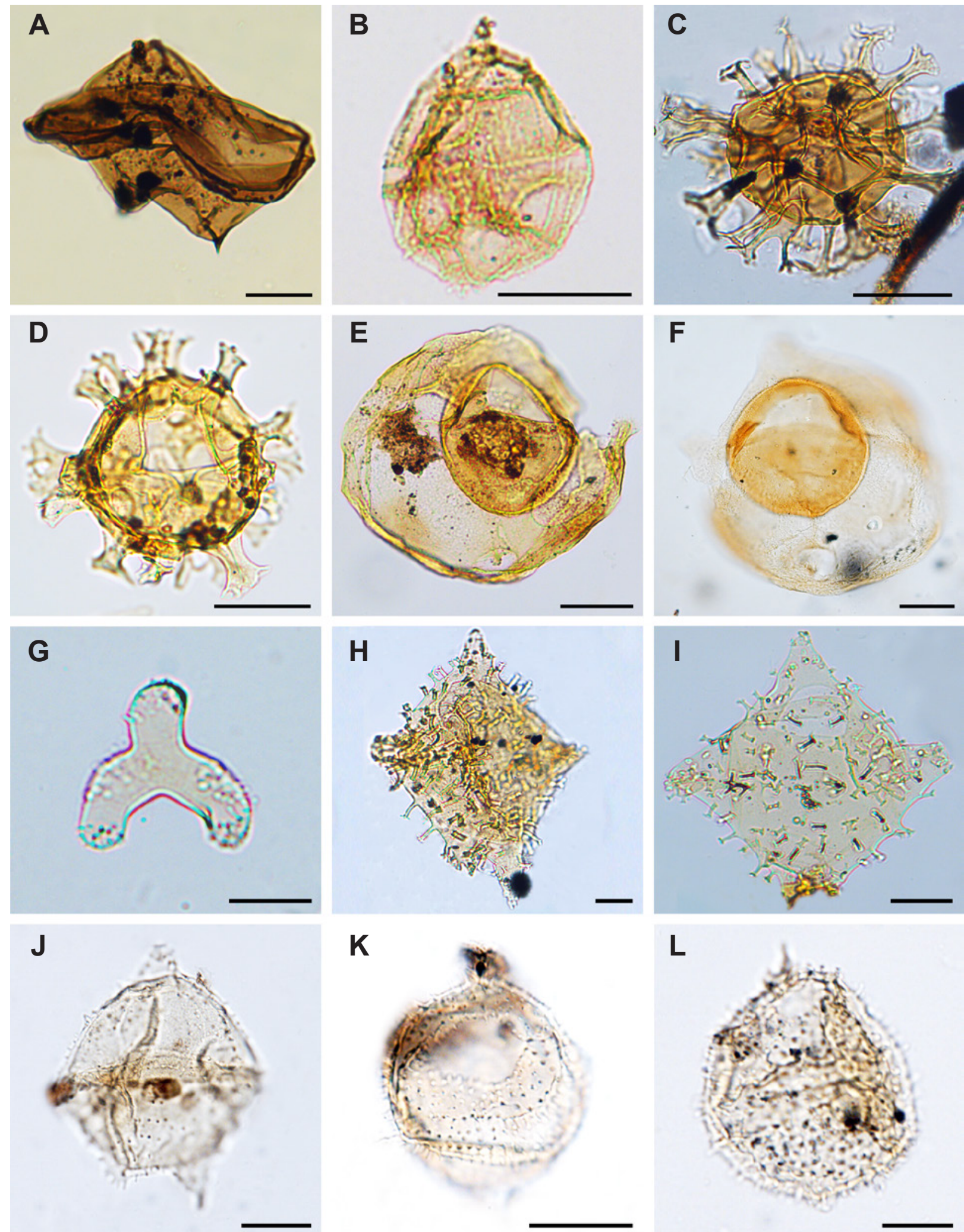

Figura 4/Figure 4. Quistes de dinoflagelados y acritarcas seleccionados del pozo Gaviotín, Cuenca Punta del Este (A-I) y de pozos de la Cuenca del Colorado (J-L). Los ejemplares se identifican mediante el número de muestra y referencias del England Finder / Selected dinoflagellate cysts and acritarch from the Gaviotín borehole, Punta del Este Basin (A-I) and from Colorado Basin wells (J-L). Specimens are identified by sample number and England Finder references. A, Phelodinium magnificum Stover \& Evitt, 1978 (1549E4, L28/2-L28/4); B, Phthanoperidinium geminatum Bujak et al., 1980 (1549B2, F26/4); C-D, Spiniferites pseudofurcatus (Klumpp, 1953) Sarjeant, 1970 emend. Sarjeant, 1981; C, (1576B, N27/3); D, (1567A, F21/4); E-F, Thalassiphora pelagica (Eisenack) Eisenack \& Gocht, 1960; E, (1476D, U13-4); F, (1576A, O25/2); G, Tritonites asteris Marshall \& Partridge, 1988 (1549A, Q27/2); H, Wetzeliella sp. 1 (1546A, V28/3); I, Wetzeliella sp. 2 (1512D, P32/4- P33/3); J, Spinidinium macmurdoense (Wilson) Lentin \& Williams, 1976 (PB 514, J53/1); K, Vozzhennikovia apertura (Wilson) Lentin \& Williams, 1976 (PB514, O10; L, Vozzhennikovia sp. cf. V. apertura (Wilson) Lentin \& Williams, 1976 (PB514, D60/0). Escalas/ Scale bars: A-F, H-L = $20 \mu \mathrm{m} ; \mathrm{G}=10 \mu \mathrm{m}$. 
Vozzhennikovia sp. cf. V. apertura, que muestra una tendencia creciente de base a tope de la sección, mientras que Spinidium macmurdoense se registra con valores muy bajos a lo largo de todo el intervalo. Deflandrea antarctica se presenta de manera frecuente a los 1476 y $1576 \mathrm{mbp}$ con muy bajos valores en los niveles intermedios. Por su parte, Senegalinium sp. tiene muy escasa representación en los niveles 1549 y 1576.

Entre las especies cosmopolitas Lingulodinium machaerophorum, Operculodinium spp., Spiniferites ramosus y $S$. pseudofurcatus se registran de manera común a frecuente en todos los niveles, con la excepción de este último que se vuelve abundante en el nivel 1567; Enneadocysta arcuata está representada en todas las asociaciones pero con valores más bajos y Adnatosphaeridium multispinosum se registra sólo en la base del intervalo, en el nivel 1576. Otras especies cosmopolitas que se destacan por su presencia en la mayor parte de las asociaciones, aunque con baja abundancia, son Thalassiphora pelagica y Turbiosphaera sp.
La distribución de especies muestra que las asociaciones están ampliamente dominadas por los taxa endémicos gonyaulacoideos Enneadocysta dictyostila y E. brevistila a lo largo de toda la sección, seguidos por las especies cosmopolitas y valores menores de las especies endémicas peridinioideas. Se registró una especie de acritarca, Tritonites asteris, en el nivel 1549.

\section{Cuenca del Colorado}

La Tabla 4 ilustra la distribución de frecuencias semicuantitativas registradas en los intervalos analizados en las siguientes perforaciones:

Pozo Ombucta-1. Guerstein \& Guler (2000) analizaron 31 muestras de cutting entre 250 y 850 metros de profundidad $(\mathrm{mbp})$. Para este estudio se re-examinaron tres muestras del intervalo 694-770 asignado previamente a una edad comprendida entre el Eoceno y el Oligoceno temprano. Se identificaron 23 especies, de las cuales 20 son cosmopolitas y

Tabla 1. Número de las muestras correspondientes a las distintas perforaciones de las cuencas Punta del Este y Colorado.

Table 1. Number of the samples corresponding to the different boreholes of the Punta del Este and Colorado basins.

\begin{tabular}{|c|c|c|c|}
\hline $\begin{array}{l}\text { Intervalo (mbp) } \\
\mathrm{N}^{\circ} \text { Muestras }\end{array}$ & Colección & Compañía/ubicación & Referencia \\
\hline \multicolumn{4}{|c|}{ CUENCA PUNTA DEL ESTE } \\
\hline \multicolumn{4}{|c|}{ Pozo Gaviotín } \\
\hline $1476-1576$ & Dpto. de Paleontología, Fac. & CHEVRON & (Daners \& Guerstein, 2004) \\
\hline (8 muestras) & Ciencias, UDELAR (FC-PP) & Offshore Uruguay & Este trabajo \\
\hline \multicolumn{4}{|c|}{ CUENCA DEL COLORADO } \\
\hline \multicolumn{4}{|c|}{ Pozo Pto Belgrano 20} \\
\hline $493-559$ & INGEOSUR & SEGEMAR & Guerstein (1990) \\
\hline (3 muestras) & Dpto. Geología UNS & Onshore & Este trabajo \\
\hline \multicolumn{4}{|c|}{ Pozo_Ombucta x-1 } \\
\hline $694-770$ & INGEOSUR & YPF & Guerstein \& Guler (2000) \\
\hline (5 muestras) & Dpto. Geología UNS & Onshore & Este trabajo \\
\hline \multicolumn{4}{|c|}{ Pozo Cx-1 } \\
\hline $1195-1320$ & INGEOSUR & PHILLIPS/AGIP & Guerstein \& Junciel (2001) \\
\hline (7 muestras) & Dpto. Geología UNS & Offshore Argentina & Este trabajo \\
\hline \multicolumn{4}{|c|}{ Pozo Pejerrey } \\
\hline $1550-1754$ & INGEOSUR & SHELL -CAPSA & $\begin{array}{c}\text { Archangelsky et al. (1997 - informe } \\
\text { inédito) }\end{array}$ \\
\hline (5 muestras) & Dpto. Geología UNS & Offshore Argentina & Este trabajo \\
\hline \multicolumn{4}{|c|}{ Pozo Cruz del Sur } \\
\hline $1700-1750$ & INGEOSUR & UNION TEXAS & Quattrocchio \& Guerstein (1994 - \\
\hline (6 muestras) & Dpto. Geología UNS & Offshore Argentina & informe inédito) \\
\hline \multicolumn{4}{|c|}{ Pozo Dx-1 } \\
\hline $960-1120$ & INGEOSUR & PHILLIPS/AGIP & Culor \& Curctoin (2000) \\
\hline (4 muestras) & Dpto. Geología UNS & Offshore Argentina & Guler \& Guerstem (2002) \\
\hline \multicolumn{4}{|c|}{ Pozo Fx-1 } \\
\hline $850-910$ & INGEOSUR & PHILLIPS/AGIP & Guler \& Guerstein (2000) \\
\hline (2 muestras) & Dpto. Geología UNS & Offshore Argentina & Guler \& Guerstein (2002) \\
\hline \multicolumn{4}{|c|}{ Pozo Estrella } \\
\hline $\begin{array}{l}1780-1840 \\
(3 \text { muestras })\end{array}$ & Micro-Strat Inc (Houston - EEUU) & $\begin{array}{l}\text { UNION TEXAS } \\
\text { Offshore Argentina }\end{array}$ & Frydlund (com. pers.) \\
\hline
\end{tabular}


Tabla 2. Lista de especies de quistes de dinoflagelados registradas en las perforaciones de la Cuenca Punta del Este y Cuenca del Colorado. La clasificación está tomada de Fensome et al. (2006), Sluijs et al. (2009) y Williams et al. (2015). La distribución latitudinal se basa en Bijl et al. (2011) y Houben et al. (2013). Abreviaturas: G, gonyaulacales; P, peridiniales.

Table 2. List of dinoflagellate cyst species registered in Punta del Este and Colorado Basins boreholes. The classification is based on Fensome et al. (2006), Sluijs et al. (2009) and Williams et al. (2015). The latitudinal distribution is based on Bijl et al. (2011) and Houben et al. (2013). Abbreviations: $\mathbf{G}$, gonyaulacales; $\mathbf{P}$, peridiniales.

\begin{tabular}{|c|c|c|c|}
\hline TAXA & Figura & Orden & Distribución \\
\hline Achilleodinium biformoides (Eisenack) Eaton, 1976 & $1 \mathrm{~A}$ & G & Cosmopolita \\
\hline Adnatosphaeridium multispinosum Williams \& Downie, 1966 & 1B & G & Cosmopolita \\
\hline Batiacasphaera baculata Drugg, 1970 & & G & Cosmopolita \\
\hline Charlesdowniea sp. 1 & $1 \mathrm{C}$ & $\mathrm{P}$ & Cosmopolita \\
\hline Charlesdowniea sp. 2 & $1 \mathrm{D}$ & $\mathrm{P}$ & Cosmopolita \\
\hline Cleistosphaeridium ancyreum (Cookson \& Eisenack) Eaton et al., 2001 & & G & Cosmopolita \\
\hline Cleistosphaeridium diversispinosum (Davey, Downie, Sarjeant \& Williams,) emend. Eaton et al., 2001 & & G & Cosmopolita \\
\hline Cleistosphaeridium placacanthum (Deflandre \& Cookson) Eaton et al., 2001 & & G & Cosmopolita \\
\hline Cleistosphaeriduim polypetellum (Islam) Stover \& Williams, 1995 & & G & Cosmopolita \\
\hline Cordosphaeridium minimum (Morgenroth) Benedek, 1972 & & G & Cosmopolita \\
\hline Cordosphaeridium sp. & $1 \mathrm{E}$ & G & Cosmopolita \\
\hline Cribroperidinium giuseppei (Morgenroth) Helenes, 1984 & $1 \mathrm{~F}$ & G & Cosmopolita \\
\hline Cribroperidinium tenuitabulatum (Gerlach) Helenes, 1984 & & G & Cosmopolita \\
\hline Deflandrea antarctica Wilson, 1967 & $1 \mathrm{G}$ & $\mathrm{P}$ & Endémica- antártica \\
\hline Deflandrea phosphoritica Eisenack, 1938 & $1 \mathrm{H}$ & $\mathrm{P}$ & Cosmopolita \\
\hline Dapsilidinium pastielsii (Davey \& Williams) Bujak et al., 1980 & & G & Cosmopolita \\
\hline Diphyes colligerum (Deflandre\& Cookson) Cookson, 1965 & $1 \mathrm{I}$ & $\mathrm{G}$ & Cosmopolita \\
\hline Emmetrocysta urnaformis (Cookson) Stover, 1975 & $1 \mathrm{~J}$ & G & Cosmopolita \\
\hline Enneadocysta arcuata (Eaton) Stover \& Williams, 1995 & $1 \mathrm{~K}, 2 \mathrm{~B}, \mathrm{C}$ & G & Cosmopolita \\
\hline Enneadocysta brevistila Fensome et al., 2006 & $1 \mathrm{~L}, 2 \mathrm{D}$ & G & Endémica-antártica \\
\hline Enneadocysta dictyostila (Menéndez) Stover \& Williams, 1995 emend. Fensome et al., 2006 & $2 \mathrm{~A}, \mathrm{E}$ & G & Endémica-antártica \\
\hline Fibrocysta bipolaris (Cookson \& Eisenack) Stover \& Evitt, 1978 & & G & Cosmopolita \\
\hline Heteraulacacysta pustulata Jan du Chêne \& Adediran, 1985 & & G & Cosmopolita \\
\hline Hystrichokolpoma rigaudiae Deflandre \& Cookson, 1955 & & G & Cosmopolita \\
\hline Hystrichokolpoma salacia Eaton, 1976 & $2 \mathrm{~F}$ & G & Cosmopolita \\
\hline Impagidinium sp. & $2 \mathrm{G}$ & G & Cosmopolita \\
\hline Lejeunecysta sp. 1 & $2 \mathrm{H}$ & $\mathrm{P}$ & Cosmopolita \\
\hline Lejeunecysta sp. 2 & 2I & $\mathrm{P}$ & Cosmopolita \\
\hline Lingulodinium machaerophorum (Deflandre \& Cookson) Wall, 1967 & $2 \mathrm{~J}$ & G & Cosmopolita \\
\hline Melitasphaeridium pseudorecurvatum (Morgenroth) Bujak et al., 1980 & & G & Cosmopolita \\
\hline Muratodinium fimbriatum (Cookson \& Eisenack) Drugg, 1970 & $2 \mathrm{~K}$ & G & Cosmopolita \\
\hline Operculodinium centrocarpum (Deflandre \& Cookson) Wall, 1967 & $2 \mathrm{~L}$ & G & Cosmopolita \\
\hline Operculodinium israelianum (Rossignol) Wall, 1967 & & G & Cosmopolita \\
\hline Phelodinium magnificum (Stanley) Stover \& Evitt, 1978 & $3 \mathrm{~A}$ & $\mathrm{P}$ & Cosmopolita \\
\hline Phthanoperidinium comatum (Morgenroth) Eisenack \& Kjellström, 1972 & & $\mathrm{P}$ & Cosmopolita \\
\hline Phthanoperidinium geminatum Bujak in Bujak et al., 1980 & 3B & $\mathrm{P}$ & Cosmopolita \\
\hline Reticulatosphaera actinocoronata (Benedek) Bujak \&Matsuoka, 1986 & & G & Cosmopolita \\
\hline Schematophora speciosa (Deflandre \& Cookson) emend. Stover, 1975 & & G & Cosmopolita \\
\hline Senegalinium sp. & & $\mathrm{P}$ & Endémica-antártica \\
\hline Spinidinium macmurdoense (Wilson) Lentin \& Williams, 1976 & $3 \mathrm{~J}$ & $P$ & Endémica-antártica \\
\hline Spiniferites membranaceous (Rossignol) Sarjeant, 1970 & & G & Cosmopolita \\
\hline Spiniferites pseudofurcatus (Klumpp) Sarjeant, 1970 emend. Sarjeant, 1981 & $3 \mathrm{C}-\mathrm{D}$ & G & Cosmopolita \\
\hline Spiniferites ramosus (Ehrenberg) Mantell, 1854 & $3 \mathrm{~J}$ & G & Cosmopolita \\
\hline Thalassiphora pelagica (Eisenack) Eisenack \& Gocht, 1960 & $3 \mathrm{E}-\mathrm{F}$ & G & Cosmopolita \\
\hline Tritonites asteris Marshall \& Partridge, 1988 & $3 \mathrm{G}$ & - & Cosmopolita \\
\hline Turbiosphaera sp. & & G & Cosmopolita \\
\hline Vozzhennikovia apertura (Wilson) Lentin \& Williams, 1976 & $3 \mathrm{~K}$ & $\mathrm{P}$ & Endémica-antártica \\
\hline Vozzhennikovia sp.cf. V. apertura (Wilson) Lentin \& Williams, 1976 & $3 \mathrm{~L}$ & $\mathrm{P}$ & Endémica-antártica \\
\hline Wetzeliella sp. 1 & $3 \mathrm{H}$ & $\mathrm{P}$ & Cosmopolita \\
\hline Wetzeliella sp. 2 & $3 \mathrm{I}$ & $\mathrm{P}$ & Cosmopolita \\
\hline
\end{tabular}


Tabla 3. Distribución (número de especímenes por muestra) de quistes de dinoflagelados y acritarcas registrados en el pozo Gaviotín, Cuenca Punta del Este.

Table 3. Distribution (number of specimens per sample) of dinoflagellates cyst species and acritarchs recorded in Gaviotín borehole, Punta del Este Basin.

\begin{tabular}{|c|c|c|c|c|c|c|c|c|}
\hline Pozo Gaviotín & 1476 & 1512 & 1521 & 1530 & 1549 & 1558 & 1567 & 1576 \\
\hline Achilleodinium biformoides & $\mathrm{R}$ & & & & MR & & & \\
\hline Adnatosphaeridium multispinosum & & & & & & & & $\mathrm{F}$ \\
\hline Cribroperidinium giuseppei & & & & & MR & & & \\
\hline Cribroperidinium tenuitabulatum & & & & & MR & & & \\
\hline Deflandrea antarctica & $\mathrm{F}$ & & $\mathrm{R}$ & & MR & MR & MR & $\mathrm{F}$ \\
\hline Deflandrea phosphoritica & & & & & MR & MR & & \\
\hline Deflandrea $\mathrm{sp}$. & MR & & MR & MR & MR & & & \\
\hline Diphyes colligerum & $\mathrm{R}$ & & & & & & & \\
\hline Emmetrocysta urnaformis & & MR & & & MR & MR & & $\mathrm{R}$ \\
\hline Enneadocysta arcuata & $\mathrm{F}$ & $\mathrm{R}$ & $\mathrm{F}$ & MR & MR & $\mathrm{R}$ & $\mathrm{F}$ & $\mathrm{R}$ \\
\hline Enneadocysta brevistila & $\mathrm{F}$ & F & $\mathrm{F}$ & $\mathrm{R}$ & MR & MR & $\mathrm{F}$ & MR \\
\hline Enneadocysta dictyostila & A & A & A & A & A & A & A & $\mathrm{A}$ \\
\hline Hystrichokolpoma salacia & $\mathrm{R}$ & & & MR & $\mathrm{R}$ & & & \\
\hline Impagidinium sp. & MR & & MR & & MR & & & \\
\hline Lejeunecysta sp. & & & & & $\mathrm{R}$ & & & \\
\hline Lejeunecysta sp.1 & & & & & & MR & & \\
\hline Lejeunecysta sp.2 & & & & & & MR & & \\
\hline Lingulodinium machaerophorum & $\mathrm{F}$ & $\mathrm{F}$ & $\mathrm{R}$ & $\mathrm{R}$ & & $\mathrm{R}$ & $\mathrm{R}$ & MR \\
\hline Muratodinium fimbriatum & & & & & MR & & & \\
\hline Operculodinium spp. & $\mathrm{C}$ & $\mathrm{F}$ & $\mathrm{F}$ & $\mathrm{R}$ & $\mathrm{F}$ & $\mathrm{R}$ & $\mathrm{C}$ & $\mathrm{F}$ \\
\hline Phelodinium magnificum & & & & & MR & & & \\
\hline Phthanoperidinium geminatum & $\mathrm{R}$ & MR & MR & & MR & & & \\
\hline Senegalinium sp. & & & & & MR & & & MR \\
\hline Spinidinium macmurdoense & MR & MR & $\mathrm{R}$ & MR & $\mathrm{R}$ & MR & $\mathrm{R}$ & \\
\hline Spiniferites pseudofurcatus & $\mathrm{C}$ & $\mathrm{R}$ & $\mathrm{F}$ & $\mathrm{R}$ & & MR & A & $\mathrm{R}$ \\
\hline Spiniferites spp. & $\mathrm{F}$ & $\mathrm{F}$ & $\mathrm{F}$ & $\mathrm{R}$ & $\mathrm{R}$ & $\mathrm{R}$ & $\mathrm{F}$ & $\mathrm{F}$ \\
\hline Thalassiphora pelagica & $\mathrm{R}$ & MR & $\mathrm{R}$ & $\mathrm{R}$ & MR & & MR & MR \\
\hline Tritonites asteris & & & & & MR & & & \\
\hline Turbiosphaera sp. & MR & MR & & MR & MR & MR & & MR \\
\hline Vozzhennikovia sp. cf. V. apertura & $\mathrm{C}$ & $\mathrm{F}$ & $\mathrm{F}$ & $\mathrm{R}$ & $\mathrm{F}$ & & $\mathrm{R}$ & MR \\
\hline Wetzeliella $\mathrm{sp} .1$ & & & & & & & & MR \\
\hline Wetzeliella sp. 2 & & & & & & MR & & MR \\
\hline
\end{tabular}

\section{Referencias}

Ejemplares por preparado:

MR: muy raro (1)

$\mathrm{R}: \operatorname{raro}(2$ a 5$)$

F: frecuente $(6$ a 20$)$

C: común $(21$ a 40$)$

A: abundante $(>40)$

resultan dominantes en todas las asociaciones. Entre los taxa de la asociación endémica-antártica, Enneadocysta dictyostila está presente en los niveles inferiores del intervalo analizado de forma frecuente y ausente en el tramo superior. Spinidinium macmurdoense se registra en los niveles intermedios (752 y 728), siendo frecuente sólo en este último. Vozzhennikovia sp. cf. $V$. apertura se encuentra en todas las muestras del intervalo analizado con valores que aumentan levemente hacia los niveles más altos (708 y 694).

Las especies con distribución cosmopolita que se registran de manera consistente y con mayor representatividad en todo el intervalo aquí estudiado son: Spiniferites ramosus, S. spp., Operculodinium spp., Lingulodinum machaerophorum y Hystrichokolpoma rigaudiae. Con frecuencias menores se registran en todos los niveles: Emmetrocysta urnaformis, Cribroperidinium tenuitabulatum y Cleistosphaeridium diversispinosum. Las especies registradas solamente en las dos asociaciones superiores del intervalo analizado (694 y 708) son Cleistosphaeridium placacanthum, Dapsilidinium pastielsii, Impagidinium sp., Lejeunecysta sp., Reticulatosphaera actinocoronata y Thalassiphora sp. cf. T. pelagica.

Pozo Puerto Belgrano 20. Entre los 40 y $630 \mathrm{mbp}$ fueron procesadas 22 muestras de cutting para palinología, de las cuales solo siete resultaron fértiles (Guerstein, 1990). Para este trabajo se re-analizaron las tres muestras provenientes del intervalo 493-559. Se reconocieron 21 especies de dinoflagelados, en su mayoría de distribución cosmopolita. 


\begin{tabular}{|c|c|c|c|c|c|c|c|}
\hline $\begin{array}{ll}5 \\
5 \\
5\end{array}$ & $\tilde{\Sigma}$ & & $\simeq$ & 工 & & $\tilde{\Sigma}$ & $\tilde{\Sigma}$ \\
\hline 点 & 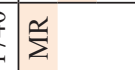 & & $\simeq$ & I & & $\simeq \cong$ & I \\
\hline 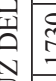 & & & $\simeq \tilde{\Sigma}$ & $\stackrel{\xi}{\Sigma}$ & & 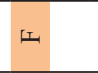 & $\Phi \simeq$ \\
\hline 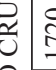 & $\simeq \cong$ & $\tilde{\Sigma}$ & & & $\stackrel{\Sigma}{\Sigma}$ & 山 & $\simeq 4$ \\
\hline $\begin{array}{l}9 \\
0 \\
0 \\
0\end{array}$ & $\stackrel{\Sigma}{2} \simeq$ & & $\simeq$ & & & $\simeq$ & $\Phi \simeq$ \\
\hline$£$ & $\stackrel{\Sigma}{2}$ & & $\simeq$ & & & $\simeq$ & $\simeq 1$ \\
\hline
\end{tabular}

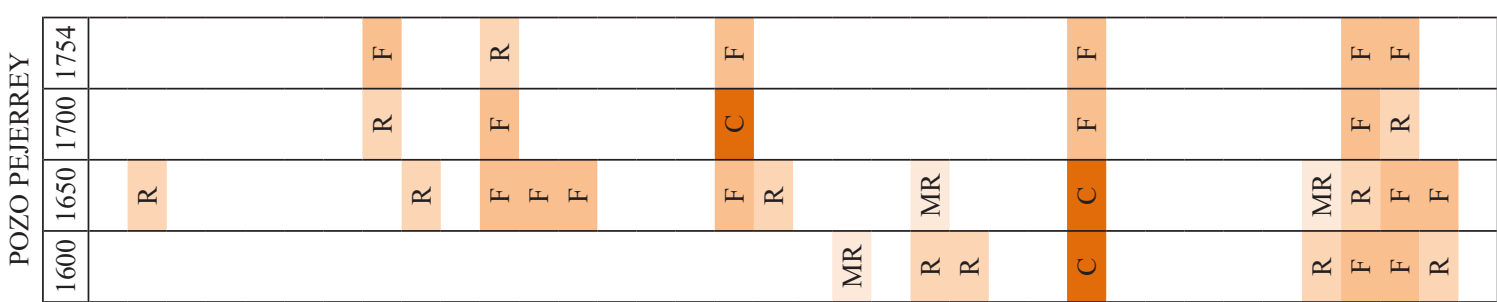

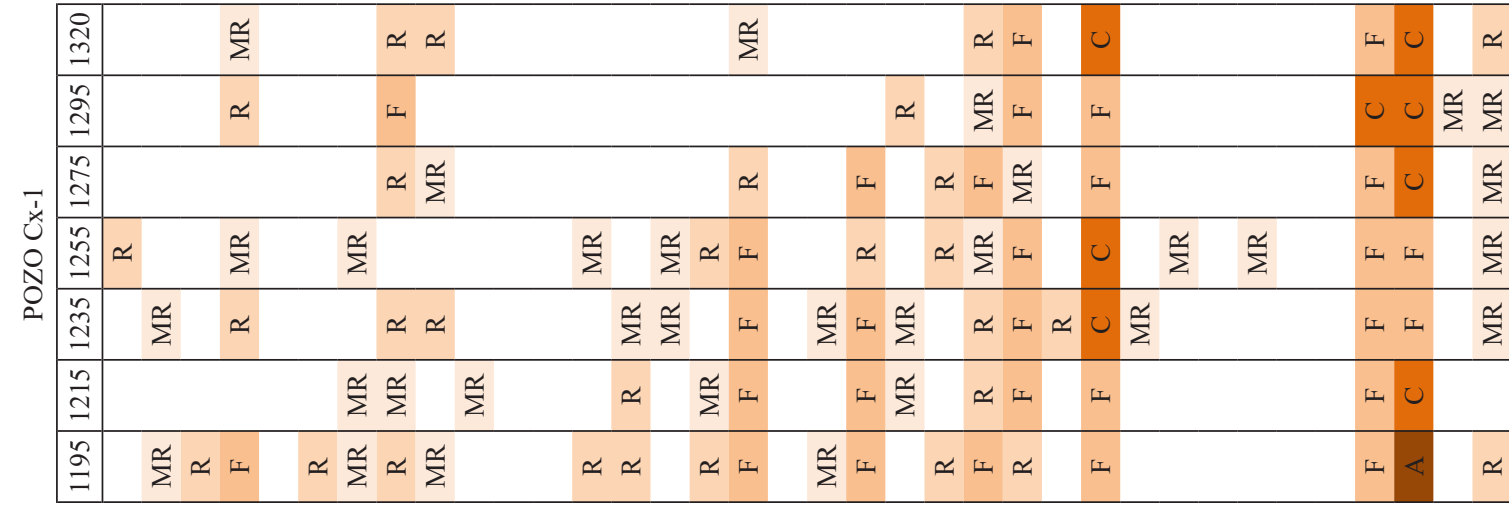

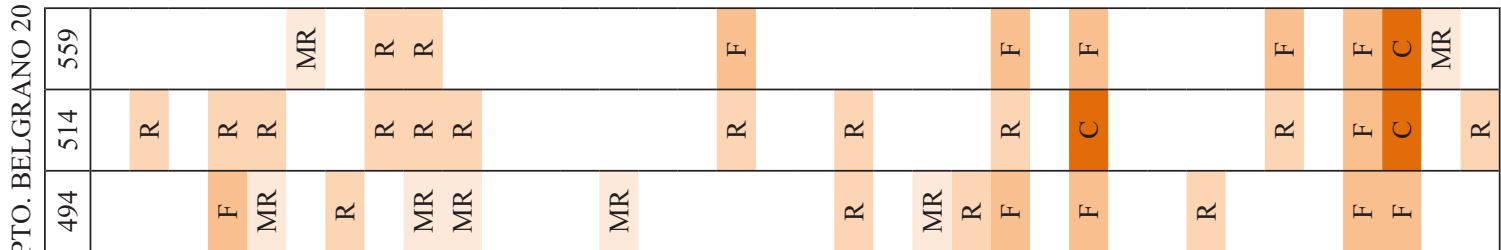


Las tres especies endémicas registradas, Vozzhennikovia sp. cf. V. apertura, Enneadocysta dicyostila y Spinidinium macmurdoense, se presentan en baja cantidad. Las dos últimas mencionadas presentan un aumento relativo hacia la base de la sección, mientras que Vozzhennikovia se registra solamente en el nivel intermedio con pocos ejemplares.

Se encuentran presentes de manera común a frecuente en todos los niveles Spiniferites ramosus, Spiniferites spp., Operculodinium spp., Lingulodinum machaerophorum y con menor representatividad Cribroperidinium tenuitabulatum. Las especies endémicas alcanzan solo un cuarto de la asociación en el nivel inferior disminuyendo progresivamente hacia el techo del intervalo analizado.

Pozo Cx-1. De las 60 muestras de cutting entre 200 y $1900 \mathrm{~m}$ de profundidad se re-analizaron para este estudio 7 muestras prevenientes del intervalo comprendido entre $1195 \mathrm{y}$ $1320 \mathrm{mbp}$., el que había sido previamente asignado al Eoceno superior-Oligoceno inferior por Guerstein \& Junciel (2001). Se reconocieron 20 especies, de las cuales 3 pertenecen a la asociación endémica-antártica, Enneadocysta dictyostila, E. brevistila y Vozzhennikovia sp. cf. V. apertura.
Enneadocysta dictyostila está presente en casi todas las asociaciones excepto en el nivel 1295, aumentando su representación hacia los niveles superiores, volviéndose frecuente. Es también hacia los niveles superiores que se registra E. brevistila, aunque con menor cantidad de ejemplares. Vozzhennikovia sp. cf. V. apertura tiene una presencia permanente en las asociaciones, aunque con muy poca cantidad de ejemplares.

Dentro de las especies con distribución cosmopolita, Spiniferites spp. es la única que se registra de manera abundante en el nivel superior (1195), y es común a frecuente en el resto de los niveles. Representadas en todos los niveles de manera común a frecuente están $S$. ramosus, Operculodinium spp. y Lingulodinum machaerophorum. Con frecuencias menores se registran en todos o la mayor parte de los niveles: Cleistosphaeridium diversispinosum, Cordosphaeridium sp., Hystrichokolpoma rigaudiae y Lejeunecysta sp.

En general las asociaciones están ampliamente dominadas por las especies de distribución cosmopolita, mientras que las especies representantes de la flora endémica-antártica muestran un aumento en el registro de su frecuencia en los niveles superiores.

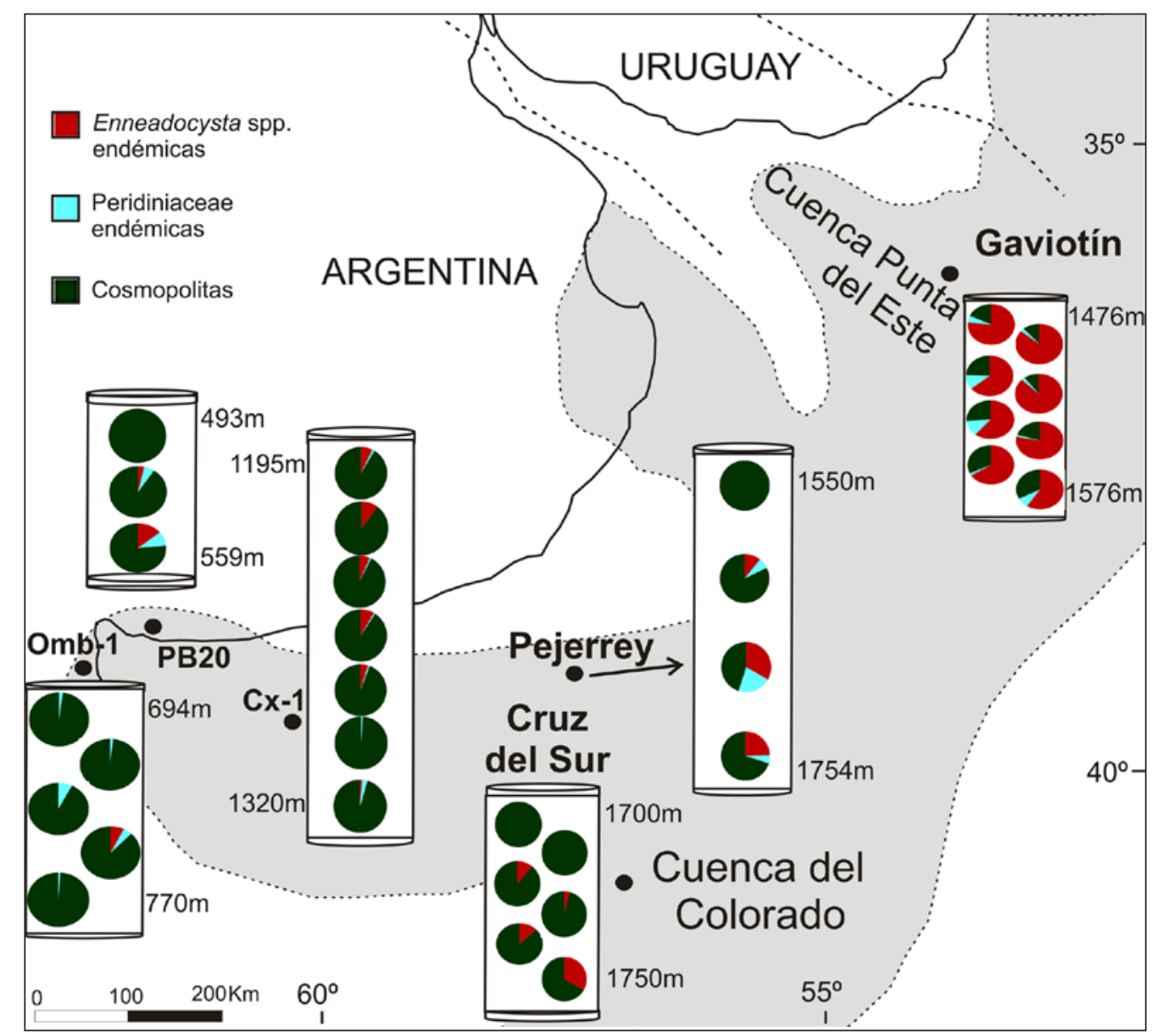

Figura 5. Distribución de las especies de Enneadocysta endémicas, quistes peridináceos endémicos y taxones cosmopolitas por pozo analizado en las cuencas Punta del Este y Colorado.

Figure 5. Distribution of endemic Enneadocysta species, endemic peridiniacean cysts and cosmopolitan taxa at whole sites in Punta del Este and Colorado basins. 
Pozo Pejerrey. Archangelsky et al. (1997) procesaron 59 muestras de cutting provenientes del intervalo estratigráfico entre 500 y 3000 metros de profundidad, de las cuales 37 resultaron fértiles. Para este trabajo se re-estudiaron cuatro muestras entre 1600 y 1754 mbp originalmente asignados al Eoceno. Se identificó un total de 16 especies, de las cuales solamente dos pertenecen a la flora endémica-antártica, Deflandrea antarctica y Enneadocysta dictyostila. Éstas se encuentran en las tres muestras inferiores del intervalo, con frecuencias más altas en los niveles medios (1650 y 1700).

Las especies con distribución cosmopolita con representatividad frecuente a común en todo el intervalo aquí estudiado son Operculodinium spp. y en menores cantidades Spiniferites ramosus y Spiniferites spp.

Las asociaciones superiores de este intervalo están francamente dominadas por las especies de distribución cosmopolita, haciéndose más importantes hacia la parte inferior de la sección, llegando a dominar en el nivel 1700.

Pozo Cruz del Sur. Quattrocchio \& Guerstein (1994) procesaron 191 muestras de cutting entre los 450 y $2200 \mathrm{~m}$ de profundidad, resultando 154 palinológicamente fértiles. Para este trabajo se consideraron seis muestras provenientes del intervalo entre 1700 y $1750 \mathrm{~m}$, las que originalmente fueron asignadas al Eoceno. Se reconocieron 12 especies, de las cuales 11 son cosmopolitas. La única especie endémica, Enneadocysta dictyostila, se registra de manera frecuente en los 1740 y $1750 \mathrm{mbp}$ y con valores muy bajos en 1720 y 1730 .

Entre las especies cosmopolitas, se registran en todos los niveles con frecuencias variables de común a rara, Lingulodinium machaerophorum, Spiniferites ramosus y Spiniferites spp.

Las especies pertenecientes a la asociación endémicaantártica están ausentes en los niveles superiores, mostrándose subordinadas a las especies de quistes de dinoflagelados de distribución cosmopolita en el resto de las asociaciones del intervalo. Sin embargo, éstas muestran un claro aumento en su representación hacia los niveles inferiores.

\section{DISCUSIÓN}

\section{Edad de las secciones estudiadas}

Las unidades estudiadas en los pozos que atraviesan el Eoceno no afloran en las cuencas de latitudes medias del Atlántico Sudoccidental por lo que toda la información disponible proviene de datos de subsuelo. El potencial de los quistes de dinoflagelados como indicadores bioestratigráficos en el Paleógeno del Hemisferio Sur se ha visto notoriamente incrementado en los últimos años a través de los estudios realizados sobre testigos de ODP e IODP en el Pacífico Sur (Bijl et al., 2013b y trabajos allí citados). Asimismo, trabajos recientes sobre secciones aflorantes en el sudoeste de la Cuenca Austral (González Estebenet et al., 2014b, 2015; Guerstein et al., 2008b, 2014) constituyen una importante fuente de información que puede ser considerada para analizar los resultados obtenidos en las cuencas de latitudes medias. En este caso, utilizamos los eventos de quistes de dinoflagelados de las especies diagnósticas basadas en la bioestratigrafía de alta resolución propuesta por Bijl et al. (2013b) para el Paleógeno del Pacífico Sur (South Pacific Dinocyst Zones, SPDZ) y las asociaciones de quistes de dinoflagelados para la transición Eoceno-Oligoceno de Sluijs et al. (2003). Los registros utilizados para elaborar dichas zonaciones están calibrados con magnetoestratigrafía y estratigrafía de isótopos estables.

En este trabajo, por tratarse de material de cutting, el análisis bioestratigráfico prioriza las últimas ocurrencias (UO) y las últimas ocurrencias comunes o abundantes (UOC - UOA) por sobre las primeras ocurrencias o bases de los registros estratigráficos, las que pueden ser utilizadas con reserva cuando es posible considerar que el evento esté representado por ejemplares in situ. La mayoría de las especies halladas en este trabajo presentan rangos estratigráficos largos, pero la combinación de eventos de dinoflagelados permite proponer edades mínimas y máximas para los distintos intervalos analizados.

Pozo Gaviotín. Es destacable que todas las asociaciones de quistes de dinoflagelados del intervalo $1476-1576 \mathrm{~m}$ presentan gran abundancia de Enneadocysta dictyostila, acompañada por Enneadocysta brevistila (Tabla 3 y Figura 5). Recientemente, Guerstein et al. (2014) estudiaron los niveles superiores de la Formación Man Aike que aflora al sur del Lago Argentino y del miembro superior de la Formación Río Turbio, analizada en los testigos de Yacimientos Carboníferos Fiscales, señalando que las asociaciones están conformadas mayoritariamente por especies endémicas-antárticas con un alto predominio de Enneadocysta dictyostila. La edad de las asociaciones de las formaciones Man Aike y Río Turbio se basa en los eventos de dinoflagelados de las zonas propuestas por Bijl et al. (2013b) para el Océano Pacífico Sur (SPDZ). Guerstein et al. (2014) complementaron estos resultados con los obtenidos a partir del análisis de foraminíferos, nanofósiles calcáreos y edades isotópicas basadas en ${ }^{87} \mathrm{Sr} r{ }^{86} \mathrm{Sr}$ (Malumián, 1990; Concheyro, 1991; Casadío et al., 2009, respectivamente) y concluyeron que los niveles caracterizados por el amplio predominio de Enneadocysta dictyostila se relacionan con las zonas de quistes de dinoflagelados SPDZ12 y SPDZ13 de Bijl et al. (2013b) en un intervalo temporal acotado entre los 42 y 39 Ma. Esta información se puede extrapolar a las cuencas de latitudes medias del Atlántico Sudoccidental permitiendo asignar la sección analizada en el Pozo Gaviotín al Eoceno medio (Luteciano superior a Bartoniano inferior). La edad mínima coincide con la presencia de Diphyes colligerum, cuyo registro más joven está datado a los 39,8 Ma (Firth et al., 2013). Asimismo, la presencia de Enneadocysta arcuata en todas las asociaciones del intervalo analizado sugiere una edad no más antigua que 41,5 Ma (Firth et al., 2013). De acuerdo con Williams et al. (2015) los registros más antiguos de Wetzeliella, caracterizado por un arqueopilo soleiforme, corresponden al Luteciano tardío. La presencia de estas especies permite confirmar la edad máxima propuesta si asumimos que los ejemplares de Wetzeliella sp.1 y Wetzeliella sp.2 se encuentran in situ. Las restantes especies halladas en el intervalo 1476-1576 m del Pozo Gaviotín son consistentes con esta edad. 
Pozo Ombucta-1. Los dos niveles inferiores del intervalo analizado en este pozo son los únicos que presentan valores frecuentes de Enneadocysta dictyostila y permiten asignarlo a una edad no menor que Bartoniano temprano (39 Ma). El intervalo entre 694 y $728 \mathrm{~m}$ no puede ser más joven que Priaboniano medio, dada la presencia de Vozzhennikovia sp. cf. $V$. apertura. Esta especie es característica de la Zona SPDZ13 (Bijl et al., 2013b) donde su registro más alto está datado a los $36 \mathrm{Ma}$. La presencia frecuente de Reticulatosphaera actinocoronata a los $694 \mathrm{~m}$, si consideramos que los ejemplares se encuentran in situ, permite determinar una edad Priaboniano tardío o menor para la parte superior del intervalo analizado. Esta especie presenta su base a los 35,3 Ma en latitudes ecuatoriales y medias del Hemisferio Sur (Williams et al., 2004).

Pozo Puerto Belgrano 20. La asociación inferior del intervalo considerado para este estudio registra la presencia frecuente de Enneadocysta dictyostila y escasos ejemplares de Cleistosphaeridium polypetellum. De acuerdo con Eaton et al. (2001), este último taxón no se registra en unidades más jóvenes que Eoceno medio. El nivel de los 514 mbp contiene Spinidininium macmurdoense, por lo que podría ser tan joven como Priaboniano temprano. Por su parte, el nivel superior (496 mbp) podría asignase al comienzo del Oligoceno temprano, dado que registra una asociación compuesta por especies exclusivamente cosmopolitas de biocrones extensos y por la presencia de Reticulatosphaera actinocoronata.

Pozo Cx-1. El intervalo entre 1195 y 1275 m tiene una muy alta representación de Enneadocysta distyostila, por lo que puede ser asignado al Luteciano superior-Bartoniano inferior. Asimismo, la presencia de Cleistosphaeridum polypetellum a los $1195 \mathrm{~m}$, cuyo biocrón se extiende desde el Eoceno inferior al Eoceno medio y de Phthanoperidinium comatum a los $1235 \mathrm{~m}$ confirma una edad no mayor a los 41.4 Ma (Firth et al., 2013). Los dos niveles inferiores (1295 y 1320) carecen de indicadores bioestratigráficos relevantes, excepto por la presencia de pocos ejemplares de la asociación endémica-antártica. De acuerdo con Bijl et al. (2011) y González Estebenet et al. (2015), asociaciones con estas características representan al Luteciano inferior de la Plataforma de Tasmania y Cuenca Austral.

Pozo Pejerrey. Las tres muestras inferiores del intervalo analizado (1650-1754 m) contienen Enneadocysta dictyostila como elemento común a frecuente y Deflandrea antarctica como frecuente. Esta asociación permite establecer una correlación con los niveles asignados al Eoceno medio, entre los 1195 y los 1225 mbp del Pozo Cx-1, el intervalo entre 514 y $559 \mathrm{~m}$ del Pozo Puerto Belgrano 20 y el Pozo Ombucta -1, entre los 755 y $770 \mathrm{mbp}$. La asociación del nivel correspondiente a los $1600 \mathrm{~m}$ presenta sólo especies cosmopolitas de biocrones extendidos, por lo que se interpreta que el Oligoceno inferior puede estar representado en los niveles por encima de los 1600 me.
Pozo Cruz del Sur. Las asociaciones del intervalo inferior, entre 172 y $1750 \mathrm{mbp}$ presentan una alta dominancia de especies cosmopolitas aunque contienen una escasa representación de Deflandrea spp. y presentan numerosos ejemplares de Enneadocysta dictyostila. Por lo mencionado en las secciones precedentemente analizadas, este intervalo puede asignarse al Eoceno medio (Luteciano superiorBartoniano superior). El tramo superior, entre 1700 y 1710 mbp no contiene elementos diagnósticos y las asociaciones de dinoflagelados están integradas en su totalidad por especies cosmopolitas, de manera semejante a lo registrado en los pozos Pejerrey y Puerto Belgrano 20.

Otros registros de la Cuenca del Colorado. Los pozos Dx-1 y Fx-1 fueron estudiados por Guler \& Guerstein (2002). En ambos se registró la presencia de elementos de la asociación endémica-antártica, pudiendo reconocerse parte del Eoceno medio a través de Enneadocysta dictyostila, especie frecuente sólo entre los 1000 y 1220 m del Pozo Dx-1. En ambas secciones se registra Spinidinium sp. cf. S. macmurdoense entre los 845 y 910 metros del Pozo Fx-1 y entre los 960 y $1000 \mathrm{~m}$ de profundidad del Pozo Dx-1, intervalos que pueden asignarse en edad al Priaboniano superior.

En el Pozo Estrella la asociación endémica-antártica fue registrada entre los 1780 y 1840 m, entre ellos Enneadocysta dictyostila (como Areosphaerium diktyoplokum) y Deflandrea cygniformis. La Zona de Conjunto Diphyes se caracteriza por la presencia de los elementos cosmopolitas diagnósticos Diphyes colligerum, Enneadocysta arcuata junto con Deflandrea phosphoritica y Cordosphaeridium fibrospinosum (Fryklund, R., com. pers.). La edad mínima de esta zona está dada por el techo de Diphyes colligerum, cuyo registro más joven está datado a los 39,8 Ma (Firth et al., 2013) mientras que la presencia de Enneadocysta arcuata en el intervalo analizado sugiere una edad no más antigua que $41,5 \mathrm{Ma}$ (Firth et al., 2013).

\section{CONSIDERACIONES FINALES}

La distribución de las especies de altas latitudes del Hemisferio Sur, originalmente definida por Wrenn \& Beckman (1982) como "Flora Trasantártica", muestra que esta asociación endémica-antártica resulta dominante en los sitios del Océano Austral en paleolatitudes mayores a los $60^{\circ} \mathrm{S}$ (Sluijs et al., 2005). Bijl et al. (2011, 2013a) analizaron las causas del endemismo y propusieron que a partir de los 50 Ma el área del Conducto de Tasmania evidencia una apertura temprana a través de pasajes someros y el flujo restringido de aguas entre los océanos Índico y Pacífico, los que habrían favorecido el desarrollo de esta asociación endémica. En cuanto a la apertura del Pasaje de Drake, Livermore et al. (2005), sobre la base de perfiles de anomalías magnéticas adyacentes al margen sudamericano en el Mar de Escocia sugirieron que la subsidencia de las plataformas continentales en el área se habría iniciado a comienzos del Eoceno medio. Estudios posteriores evidenciaron la existencia de dos pasajes someros bien definidos a partir de los $41 \mathrm{Ma}$ 
(Eagles et al., 2006; Scher \& Martin, 2006; Livermore et al., 2007). González Estebenet et al. (2014a) compararon las asociaciones de quistes de dinoflagelados dominadas por la asociación endémica durante el Eoceno medio al norte y al sur del actual Pasaje de Drake y concluyeron que la historia de la apertura de dicho pasaje habría sido similar a la descripta para el Conducto de Tasmania por Bijl et al. (2013a).

Los registros analizados en las cuencas Punta del Este y Colorado revelan la presencia de ciertos miembros de esta asociación endémica-antártica en la mayoría de pozos estudiados. Teniendo en cuenta que la velocidad promedio con la que se trasladan efectivamente los dinoflagelados modernos en el estado móvil es de aproximadamente $3 \times 10^{-4}$ $\mathrm{m} / \mathrm{s}$ (Smayda, 2002), resulta evidente que la distribución de los mismos depende principalmente de los patrones de circulación oceánica superficial, entre otros factores.

El sistema climático terrestre se comporta de manera tal que la circulación oceánica superficial responde a los patrones de circulación atmosférica (Siedler et al., 2001; Marshall \& Plumb, 2008). Huber et al. (2004) combinaron los resultados de experimentos de modelos acoplados de circulación general (CGCM) con datos de microfósiles de testigos ODP sobre las plataformas de Tasmania. Los resultados del modelo muestran amplios giros en sentido horario alrededor de la Antártida para el Eoceno medio a tardío y explica los distintos grados de endemismo evidenciados por los patrones de distribución de quistes de dinoflagelados como indicadores indirectos (proxy data). Estos giros se intensifican en sus bordes occidentales produciendo corrientes relativamente frías, dirigidas hacia el Norte a lo largo del margen oriental de Australia y de Sudamérica.

La distribución de los quistes de dinoflagelados en cuencas de latitudes medias de la Plataforma del Océano Atlántico Sudoccidental puede explicarse por un mecanismo dinámico similar al propuesto por Huber et al. (2004) para el Pacífico Sur e Índico. En nuestro caso, el cierre parcial del Pasaje de Drake induciría la formación de un giro subpolar ciclónico en el Atlántico Sudoccidental (Proto-Giro de Weddell) cuya Corriente de Borde Oeste iniciada en el talud de la plataforma oeste antártica, seguiría una trayectoria similar a la actual Corriente de Malvinas sobre el talud patagónico. Resultados de modelos numéricos (Matano \& Palma, 2008; Palma et al., 2008; Combes \& Matano, 2014) sustentados por observaciones directas (Piola et al., 2010) indican que una corriente de esta naturaleza inyecta aguas oceánicas profundas hacia la plataforma adyacente, particularmente en la zona externa, e incrementa el flujo de plataforma en la misma dirección. De manera análoga, las aguas circum-antárticas y parte de la asociación endémica habrían sido conducidas hacia el norte por medio de este mecanismo. Esta hipótesis explica que las mayores abundancias de especies endémicas se registren en los pozos ubicados en las partes más externas de las cuencas analizadas (pozos Gaviotín, Pejerrey y Cruz del Sur). Por el contrario, las asociaciones de los pozos del sector interno de la Cuenca del Colorado están ampliamente dominados por especies cosmopolitas y escasas especies endémicas peridinioideas (Figura 5).
Es importante destacar que Röhl et al. (2004) asociaron los altos porcentajes de Enneadocysta spp. en el Eoceno medio de la plataforma de Tasmania con importantes concentraciones de carbonato de calcio, mientras que los máximos de Deflandrea spp. y otros quistes endémicos peridinioideos coinciden con los intervalos pobres en carbonato de calcio y ricos en nutrientes. Sobre la base de esta relación Röhl et al. (2004) propusieron que las altas frecuencias de Enneadocysta reflejan aguas relativamente más cálidas, en áreas de plataforma externa, mientras que los máximos porcentajes de Deflandrea se vinculan con ambientes someros caracterizados por aguas frías y eutróficas. Esta comparación explica que Enneadocysta dictyostila sea la especie endémica-antártica que mejor se haya adaptado a las aguas de mayor temperatura de latitudes medias, ya que es la única que tolera aguas relativamente templadas (Figura 5). Asimismo, es la que se desarrolla en áreas de plataforma externa, lo que explica que la escasa o nula representación en las asociaciones de los pozos de la parte interna de la Cuenca del Colorado.

Durante el Eoceno tardío los componentes endémicos en los distintos sitios de la Plataforma del Océano Atlántico Sudoccidental fueron reemplazados por asociaciones más diversas con indicadores de aguas frías y especies típicamente oceánicas, con un incremento de quistes de dinoflagelados heterotróficos modernos de la familia Protoperidiniaceae (Guerstein et al., 2008b; Houben et al., 2011; González Estebenet et al., 2015). La apertura y profundización del Conducto de Tasmania y el Pasaje de Drake y el subsecuente desarrollo de una incipiente Corriente Circumpolar Antártica durante el Oligoceno produjo la disrupción de los giros subpolares y la extinción de las especies endémicas-antárticas (Bijl et al., 2011; Houben et al., 2013). Esta tendencia se observa claramente en las asociaciones de los niveles superiores de algunos de los pozos considerados para este estudio (pozos Ombucta-1, Puerto Belgrano 20, Cruz del Sur y Pejerrey), lo que evidencia el cambio en los patrones de circulación oceánica en la transición Eoceno-Oligoceno.

\section{AGRADECIMIENTOS}

Las autoras desean agradecer a la Administración Nacional de Combustibles, Alcohol y Portland (ANCAP), empresa que cedió las muestras del Pozo Gaviotín, Union Texas por las muestras del pozo Cruz del Sur y Shell por las muestras del pozo Pejerrey. A M. Arai (Brasil) por el procesamiento de muestras del Pozo Gaviotín y O. Cárdenas (Argentina) por el procesamiento de las muestras de la Cuenca del Colorado. Agradecemos a M. Arai y a un revisor anónimo por sus valiosas sugerencias como árbitros de la revista, que ayudaron a mejorar el manuscrito. El presente trabajo fue financiado por proyectos del Consejo Nacional de Investigaciones Científicas y Técnicas (PIP: 112-201101-00566) y Universidad Nacional del Sur (PGI 24/H125), y por el proyecto de Dedicación Total (Universidad de la República Oriental del Uruguay) de G. Daners. 


\section{REFERENCIAS}

Archangelsky, S.; Quattrocchio, M. \& Guerstein, G.R. 1997. Pejerrey x-1 offshore well, Palynological Report. Buenos Aires, Shell Capsa, 19 p.

Barker, P.F.; Diekmann, B. \& Escutia, C. 2007. Onset of Cenozoic Antarctic glaciation. Deep-Sea Research II, 54:2293-2307. doi:10.1016/j.dsr2.2007.07.027

Benedek, P.N. 1972. Phytoplanktonten aus dem Mittelund Oberoligozän von Tönisberg (Niederrheingebiet). Palaeontographica, Abteilung B, 137:1-71.

Bijl, P.K.; Bendle, J.A.P; Bohaty, S.M.; Pross, J.; Schouten, S.; Tauxe, L.; Stickley, C.E.; McKay, R.M.; Röhl, U.; Olney, M.; Sluijs, A.; Escutia, C.; Brinkhuis, H. \& Expedition 318 Scientists. 2013a. Eocene cooling linked to early flow across the Tasmanian Gateway. Proceedings of the National Academy of Sciences, 110:9645-9650. doi:10.1073/pnas. 1220872110

Bijl, P.K.; Houben, A.J.; Schouten, S.; Bohaty, S.M.; Sluijs, A.; Reichart, G.; Damsté J.S. \& Henk Brinkhuis, H. 2010. Transient Middle Eocene atmospheric $\mathrm{CO}_{2}$ and temperature variations. Science, 330:819-821. doi:10.1126/science.1193654

Bijl, P.K.; Pross, J.; Warnaar, J.; Stickley, C.E.; Huber, M.; Guerstein, R.; Houben, A.J.P.; Sluijs, A.; Visscher H. \& Brinkhuis, H. 2011. Environmental forcings of Paleogene Southern Ocean dinoflagellate biogeography. Paleoceanography, 26:PA1202. doi:10.1029/2009PA001905

Bijl, P.K.; Schouten, S.; Brinkhuis, H.; Sluijs, A.; Reichart, G.J. \& Zachos, J.C. 2009. Early Palaeogene temperature evolution of the Southwest Pacific Ocean. Nature, 461:776-779. doi:10.1038/ nature08399

Bijl, P.K.; Sluijs A. \& Brinkhuis H. 2013b. A magneto-and chemostratigraphically calibrated dinoflagellate cyst zonation of the early Palaeogene South Pacific Ocean. Earth-Science Reviews, 124:1-31. doi:10.1016/j.earscirev.2013.04.010

Bohaty, S.M. \& Zachos, J.C. 2003. Significant Southern Ocean warming event in the late middle Eocene. Geology, 31:10171020. doi:10.1130/G19800.1

Bohaty, S.M.; Zachos, J.C.; Florindo, F. \& Delaney, M.L. 2009. Coupled greenhouse warming and deep-sea acidification in the middle Eocene. Paleoceanography, 24:PA2207. doi:10.1029/2008PA001676

Brinkhuis, H.; Munsterman, D.M.; Sengers, S.; Sluijs, A.; Wanaar, J. \& Williams, G.L. 2003a. Late Eocene to Quaternary dinoflagellate cysts from ODP Site 1168, off western Tasmania. In: N.F. Exon; J.P. Kennett \& M.J. Malone (eds.) Proceedings of the Ocean Drilling Program, College Station, Ocean Drilling Program, p. 1-36. (Scientific Results 189). doi:10.2973/odp. proc.sr.189.105.2003

Brinkhuis, H.; Sengers, S.; Sluijs, A.; Warnaar, J. \& Williams, G.L. 2003b. Latest Cretaceous to earliest Oligocene, and Quaternary dinoflagellate cysts from ODP Site 1172, East Tasman Plateau. In: N.F. Exon; J.P. Kennett \& M.J Malone (eds.) Proceedings of the Ocean Drilling Program, College Station, Ocean Drilling Program, p. 1-48. (Scientific Results 189). doi:10.2973/odp. proc.sr.189.106.2003

Bujak, J.P.; Downie, C.; Eaton, G.L. \& Williams, G.L. 1980. Dinoflagellate cysts and acritarchs from the Eocene of southern England. Special Papers in Palaeontology, 24:1-100.

Bujak, J.P. \& Matsuoka, K. 1986. Taxonomic reallocation of Cenozoic dinoflagellate cysts from Japan and the Bering Sea. Palynology, 10:235-241.

Casadío, S.; Griffin, M.; Marenssi, S.A.; Net, L.; Parras, A.; Rodriguez Raising, M. \& Santillana, S. 2009. Paleontology and sedimentology of Middle Eocene rocks in Lago Argentino area, Santa Cruz Province, Argentina. Ameghiniana, 46:27-47.

Cocozza, C.D. \& Clarke, C.M. 1992. Eocene microplankton from La Meseta Formation, northern Seymour Island. Antarctic Science, 4:355-362. doi:10.1017/S0954102092000506

Combes, V. \& Matano, R.P. 2014. A two-way nested simulation of the oceanic circulation in the Southwestern Atlantic. Journal of Geophysical Research, 119:731-756. doi:10.1002/2013JC009498

Concheyro, A. 1991. Nanofósiles calcáreos de la Formación Man Aike (Eoceno, sudeste del Lago Cardiel) Santa Cruz, Argentina. Ameghiniana, 28:385-399.

Conti, B. 2015. Sistemas petroliferos especulativos da Bacia de Pelotas (Offshore Uruguay). Universidade Estadual Paulista, Tesis de maestría, $150 \mathrm{p}$.

Cookson, I.C. 1965. Cretaceoous and Tertiary microplankton from south-eastern Australia. Prceedings of the Royal Society of Victoria, 78:85-93.

Daners, G. \& Guerstein, G.R. 2004. Dinoflagelados del Maastrichtiense-Paleógeno en la Formación Gaviotín, Cuenca Punta del Este. In: G. Veroslavsky; M. Ubilla \& S. Martínez (eds.) Cuencas Sedimentarias del Uruguay, Geología, Paleontología y Recursos naturales - Cenozoico, Facultad de Ciencias, Universidad de La República, p. 37-62.

Davey, R.J. 1969. The evolution of certain Upper Cretaceous hystrichospheres from South Africa. Palaeontologia Africana, 12: $25-51$.

Deflandre, G. \& Cookson, I.C. 1955. Fossil microplankton from Australian Late Mesozoic and Tertiary sediments. Australian Journal of Marine and Freshwater Research, 6:242-313.

Drugg, W.S. 1970. Some new genera, species, and combinations of phytoplankton from the Lower Tertiary of the Gulf Coast, U.S.A. In: THE NORTH AMERICAN PALEONTOLOGICAL CONVENTION, 1, 1969. Proceedings, Chicago, p. 809-843.

Eagles, G.; Livermore, R. \& Morris, P. 2006. Small basins in the Scotia Sea: the Eocene Drake passage gateway. Earth and Planetary Science Letters 242:343-353. doi:10.1016/j. epsl.2005.11.060

Eaton, G.L. 1971. A morphogenetic series of dinoflagellate cysts from the Bracklesham Beds of the Isle of Wight, Hampshire, England. In: Farinacci, A. (ed.) Proceedings of the 2nd Planktonic Conference, Edizioni Tecnoscienza, Rome, p. 355-379.

Eaton, G.L. 1976. Dinoflagellate cysts from the Bracklesham Beds (Eocene) of the Isle of Wight, southern England. British Museum (Natural History) Geology Bulletin, 26:227-332.

Eaton, G.L.; Fensome, R.A.; Riding, J.B. \& Williams, G.L. 2001. Re-evaluation of the status of the dinoflagellate cyst genus Cleistosphaeridium. Neues Jahrbuch für Geologie und Paläontologie, Abhandlungen, 219:171-205.

Eisenack, A. 1938. Die Phosphoritknollen der Bernsteinformation als Überlieferer tertiären Planktons. Schriften der PhysikalischÖkonomischen Gesellschaft zu Königsberg, 70:181-188.

Eisenack, A. \& Gocht, H. 1960. Neue Namen für einige Hystrichosphären der Bernsteinformation Ostpreussens. Neues Jahrbuch für Geologie und Paläontologie, Monatshefte, 11:511-518.

Eisenack, A. \& Kjellström, G. 1972. Katalog der Fossilen Dinoflagellaten, Hystrichosphären und Verwandten Mikrofossilien. Stuttgart, Schweizerbart'sche Verlagsbuchhandlung, 1132 p.

Fensome, R.A.; Guerstein, G.R. \& Williams, G.L. 2006. New insights on the Paleogene dinoflagellate cyst genera Enneadocysta and Licracysta gen. nov.: new insights based on material from offshore eastern Canada and southern Argentina. Micropaleontology, 52:385-410. doi:10.2113/gsmicropal.52.5.385 
Fensome, R.A.; MacRae, R.A. \& Williams, G.L. 2008. DINOFLAJ2, Version 1. Reston, American Association of Stratigraphic Palynologists.

Firth, J.V.; Eldrett, J.S.; Harding, I.C.; Coxall, H.K. \& Wade, B.S. 2013. Integrated biomagnetochronology for the Palaeogene of ODP Hole 647A: implications for correlating palaeoceanographic events from high to low latitudes. In: L. Jovane; E. HerreroBervera; L.A. Hinnov \& B.A. Housen (eds.) Magnetic Methods and the Timing of Geological Processes, London, Geological Society, p. 29-78. (Special Publications 373).

Franke, D.; Neben, S.; Ladage, S.; Schreckenberger, B. \& Hinz, K. 2007. Margin segmentation and volcano-tectonic architecture along the volcanic margin off Argentina/Uruguay, South Atlantic. Marine Geology, 244:46-67. doi:10.1016/j.margeo.2007.06.009

Franke, D.; Neben, S.; Schreckenberger, B.; Schulze, A.; Stiller, M. \& Kravczyk, C. 2006. Crustal structure across the Colorado Basin, offshore Argentina. Geophysical Journal International, 165:850-864. doi:10.1111/j.1365-246X.2006.02907.x

Fryklund, R.; Marshall, A. \& Steves, J. 1996. La Cuenca del Colorado. In: V.A. Ramos \& M.A. Turic (eds.) Geología y Recursos Naturales de la Plataforma Continental Argentina, Relatorio del $13^{\circ}$ Congreso Geológico Argentino y $3^{\circ}$ Congreso de Exploración de Hidrocarburos, Association Geologica Argentina, p. 135-158.

Ghidella, M.E.; Schreckenberger, B.; Paterlini, C.M. \& Abraham, D.A. 2006. Anomalías magnéticas en el margen argentino. In: REUNIÓN DE TECTÓNICA, 13, 2006. Actas, San Luis, CD-ROM, 4 p.

Gladczenko, T.P.; Hinz, K.; Eldholm, O.; Meyer, H.; Neben, S. \& Skogseid, J. 1997. South Atlantic volcanic margins. Journal of the Geological Society, 154:465-470. doi:10.1144/ gsjgs. 154.3.0465

González Estebenet, M.S.; Guerstein, G.R. \& Alperin, M.I. 2014a. Dinoflagellate cyst distribution during the middle Eocene in the Drake Passage area: paleoceanographic implications. Ameghiniana, 51:500-509. doi:10.5710/AMGH.06.08.2014.2727

González Estebenet, M.S.; Guerstein, G.R. \& Rodríguez Raising, M.E. 2014b. Middle Eocene Dinoflagellate cysts from Santa Cruz Province, Argentina: Biostratigraphy and Paleoenvironment. Review of Palaeobotany and Palynology, 211:55-65. doi:10.1016/j.revpalbo.2014.09.002

González Estebenet, M.S.; Guerstein, G.R. \& Casadío, S.A. 2015. Estudio bioestratigráfico y paleoambiental de la Formación Río Turbio (Eoceno medio - superior) en el sudoeste de Patagonia (Argentina) basado en quistes de dinoflagelados. Revista Brasileira de Paleontologia, 18:429-442. doi:10.4072/ rbp.2015.3.08

Greenwood, D.R. \& Wing, S.L. 1995. Eocene continental climates and latitudinal temperature gradients. Geology, 23:1044-1048. doi:10.1130/0091-7613(1995)023<1044:ECCALT>2.3.CO;2

Guerstein, G.R. 1990. Palinología Estratigráfica del Terciario de la Cuenca del Colorado, República Argentina. Parte III: Estudio sistemático y estadístico de la perforación Puerto Belgrano 20. Revista Española de Micropaleontología, 22:459-480.

Guerstein, G.R.; Brinkhuis, H., \& Daners, G. 2008a. Eocene circulation and dinoflagellate distribution in the Southwestern Atlanctic Ocean. In: REUNIÓN ARGENTINA DE SEDIMENTOLOGÍA, 12, 2008. Actas, Buenos Aires, p. 81.

Guerstein, G.R. \& Daners, G. 2010. Distribución de Enneadocysta (Dinoflagellata) en el Paleógeno del Atlántico Sudoccidental: implicancias paleoceanográficas. Ameghiniana, 47:461-478. doi:10.5710/AMGH.v47i4.5

Guerstein, G.R.; González Estebenet, M.S.; Alperin M.I.; Casadío S.A. \& Archangelsky, S. 2014. Correlation and paleoenvironments of middle Paleogene marine beds based on dinoflagellate cysts in southwestern Patagonia, Argentina. Journal of South American Earth Sciences, 52:166-178. doi:10.1016/j.jsames.2014.02.011

Guerstein G.R.; González Estebenet, M.S.; Ferreira, E.P.; Premaor, E.; Daners, G.; Amenábar, C.R.; \& Palma, E.D. 2015. Paleocirculación en el Océano Atlántico Sudoccidental durante el Eoceno medio. In: JORNADAS NACIONALES DE CIENCIAS DEL MAR, 9, 2015. Libro de Resúmenes, Ushuaia, p. 267.

Guerstein, G.R. \& Guler, M.V. 2000. Bioestratigrafía basada en quistes de dinoflagelados del Eoceno-Mioceno del pozo (YPF) Ombucta x-1, Cuenca del Colorado, Argentina. Ameghiniana, 37:81-90.

Guerstein, G.R.; Guler, M.V.; Williams, G.L.; Fensome, R.A. \& Chiesa, J.O. 2008b. Mid Palaeogene dinoflagellate cysts from Tierra del Fuego, Argentina: biostratigraphy and palaeoenvironments. Journal of Micropalaeontology, 27:75-94. doi:10.1144/jm.27.1.75

Guerstein, G.R.; Guler, M.V.; Brinkhuis, H. \& Warnaar, J. 2010. Mid Cenozoic Palaeoclimatic and Palaeoceanographic trends in the Southwest Atlantic Basins, a Dinoflagellate view. The Paleontology of Gran Barranca. In: R.H. Madden; A.A. Carlini; M.G. Vucetich \& R.F. Kay (eds.) The Paleontology of Gran Barranca: Evolution and Environmental Change through the Middle Cenozoic of Patagonia, Cambridge University Press, p. 398-409.

Guerstein, G.R \& Junciel, G.L. 2001. Quistes de dinoflagelados del Cenozoico de la Cuenca del Colorado, Argentina. Ameghiniana, 38:299-316.

Guler, M.V. \& Guerstein, G.R. 2002. Bioestratigrafía del OligocenoPlioceno Temprano de la Cuenca del Colorado (Argentina), basada en quistes de dinoflagelados. Revista Española de Micropaleontología, 34:359-371.

Heine, C.; Zoethout, J. \& Muller, D. 2013. Kinematics of the South Atlantic rift. Solid Earth, 4:215-253. doi:10.5194/se-4-215-2013

Helenes, J. 1984. Morphological analysis of Mesozoic-Cenozoic Cribroperidinium (Dinophyceae), and taxonomic implications. Palynology, 8:107-137. doi: 10.1080/01916122.1984.9989273

Hinz, K.; Neben, S.; Schreckenberger, B.; Roeser, H.A.; Block, M.; Gonzalvez de Souza, K. \& Meyer, H. 1999. The Argentine continental margin north of $48^{\circ} \mathrm{S}$ : sedimentary successions, volcanic activity during breakup. Marine and Petroleum Geology, 16:1-25. doi:10.1016/S0264-8172(98)00060-9

Houben, A.J.P.; Bijl, P.K.; Guerstein, R.G.; Sluijs, A. \& Brinkhuis, H. 2011. Malvinia escutiana, a new biostratigraphically important Oligocene dinoflagellate cyst from the Southern Ocean. Review of Palaeobotany and Palynology, 165:175-182. doi:10.1016/j. revpalbo.2011.03.002

Houben, A. J.; Bijl, P.K.; Pross, J.; Bohaty, S.M.; Passchier, S.; Stickley, C.E.; Röhl U.; Sugisaki S.; Tauxe L.; Flierdt T.; Olney M.; Sangiorgi F.; Sluijs A.; Escutia C.; Brinkhuis H. \& Expedition 318 Scientists. 2013. Reorganization of Southern Ocean plankton ecosystem at the onset of Antarctic glaciations. Science, 340:341-344. doi:10.1126/science.1223646

Huber, M.; Brinkhuis, H.; Stickley, C.E.; Döös, K.; Sluijs A.; Warnaar, J.; Schellenberg, S.A. \& Williams, G.L. 2004. Eocene circulation of the Southern Ocean: Was Antarctica kept warm by subtropical waters? Paleoceanography, 19:PA4026. doi:10.1029/2004PA001014

Jan du Chêne, R. \& Adediran, S.A. 1985. Late Paleocene to Early Eocene dinoflagellates from Nigeria. Cahiers de micropaléontologie, Centre nationale de la recherche scientifique, 1984-3:5-38. 
Lagabrielle, Y.; Goddéris, Y.; Donnadieu, Y.; Malavieille, J. \& Suarez, M. 2009. The tectonic history of Drake Passage and its possible impacts on global climate. Earth and Planetary Science Letters, 279:197-211. doi:10.1016/j.epsl.2008.12.037

Lentin, J.K. \& Williams, G.L. 1976. A monograph of fossil peridinioid dinoflagellate cysts. Bedford Institute of Oceanography, Report Series, 16:1-237.

Levy, R.H. \& Hardwood, D.M. 2000. Tertiary marine palynomorphs from the McMurdo Sound erratics, Antarctica. Antarctic Research Series, 76:183-242.

Livermore, R.; Nankivell, A.; Eagles, G. \& Morris, P. 2005. Paleogene opening of Drake Passage. Earth Planetary Science Letters, 236:459-470. doi:10.1016/j.epsl.2005.03.027

Livermore, R.; Hillenbrand, C.D.; Meredith, M. \& Eagles, G. 2007. Drake Passage and Cenozoic climate: an open and shut case? Geochemistry, Geophysics, Geosystems, 8:Q01005. doi:10.1029/2005GC001224

MacPhail, M.K. \& Truswell, E.M. 2004. Palynology of Site 1166, Prydz Bay, East Antarctica. In: A.K. Cooper; P.E. O'Brien \& C. Richter (eds.) Proceedings of the Ocean Drilling Program, College Station, Ocean Drilling Program, p. 1-38. (Scientific Results 188).doi:10.2973/odp.proc.sr.188.013.2004

Malumián, N. 1990. Foraminíferos de la Formación Man Aike (Eoceno, Sureste Lago Cardiel) Provincia de Santa Cruz. Revista de la Asociación Geológica Argentina, 45:365-385.

Malumián, N. \& Náñez, C. 2011. Los foraminíferos de la provincia de Santa Cruz. Su significado geológico. In: M.J. Haller (ed.) Geología y Recursos Naturales de Santa Cruz, Relatorio del $15^{\circ}$ Congreso Geológico Argentino, Asociación Geológica Argentina, p. 481-494.

Mantell, G.A. 1854. The Medals of Creation; or First Lessons in Geology and the Study of Organic Remains. 2a ed. London, Henry G. Bohn, 930 p.

Mao, S. \& Mohr, B.A.R. 1995. Middle Eocene dinocysts from Bruce Bank (Scotia Sea, Antarctica) and their paleoenvironmental and paleogeographic implications. Review of Palaeobotany and Palynology, 86:235-263. doi:10.1016/0034-6667(94)00138-A

Marshall, N.G. \& Partridge, A.D. 1988. The Eocene acritarch Tritonites gen. nov. and the age of the Marlin Channel, Gippsland Basin. Memoir of the Association of Australasian Palaeontologists, 5:239-257.

Marshall, J. \& Plumb, R.A. 2008. Atmosphere, Ocean and Climate Dynamics: An Introductory Text. Cambridge, Academic Press, 344 p.

Matano, R.P. \& Palma, E.D. 2008. The Upwellling of Downwelling Currents. Journal of Physical Oceanography, 38:2482-2500. doi:10.1175/2008JPO3783.1

Morales, E. 2013. Evoluçao Tectônica e Estratigráfica das Bacias da Margem Continental do Uruguai. Universidade Estadual Paulista, Tesis doctoral, $167 \mathrm{p}$.

Moulin, M.; Aslanian, D. \& Olivet, J.L. 2005. Geological constraints on the evolution of the Angolan margin based on reflection and refraction seismic data (ZaïAngo Project). Geophysical Journal International, 162:793-810. doi:10.1111/j.1365$246 X .2005 .02668 . x$

Palma, E.D.; Matano, R.P. \& Piola, A.R. 2008. A numerical study of the Southwestern Atlantic Shelf circulation: stratified ocean response to local and offshore forcing. Journal of Geophysical Research, 113:C11010. doi:10.1029/2007JC004720

Piola, A.R.; Martinez Avellaneda, N.; Guerrero, R.A.; Jardon, F.P.; Palma, E.D. \& Romero, S.I. 2010. Malvinas-slope water intrusions on the northern Patagonia continental shelf. Ocean Science, 6:345-359.
Pöthe de Baldis, E.D. 1966. Microplancton del Terciario de Tierra del Fuego. Ameghiniana, 4:219-228.

Quattrocchio, M. \& Guerstein, G.R. 1994. Palynological analysis of Cruz del Sur -1 Borehole. Colorado Basin, Argentina. Houston, Union Texas Petroleum Corporation, 25 p. (Informe técnico).

Röhl, U.; Brinkhuis, H.; Stickley, C.E.; Fuller, M.; Schellenberg, S.A.; Wefer, G. \& Williams, G.L. 2004. Sea level and astronomically induced environmental changes in middle and late Eocene sediments from the East Tasman Plateau. In: N.F. Exon; M. Malone \& J.P. Kennett (eds.) The Cenozoic Southern Ocean: Tectonics, Sedimentation, and Climate Change Between Australia and Antarctica, Washington, American Geophysical Union, p. 127-151. (Monograph Series 151). doi:10.1029/151GM09

Sarjeant, W.A.S. 1970. The genus Spiniferites Mantell, 1850 (Dinophyceae). Grana, 10:74-78.

Sarjeant, W.A.S. 1981. A restudy of some dinoflagellate cyst holotypes in the University of Kiel Collections. II. The Eocene holotypes of Barbara Klumpp (1953); with a revision of the genus Cordosphaeridium Eisenack, 1963. Meyniana, 33:97-132.

Scher, H.D. \& Martin, E.E. 2006. Timing and Climatic Consequences of the Opening of Drake Passage. Science, 312:428-430. doi:10.1126/science.1120044

Sexton, P.F.; Wilson, P.A. \& Norris, R.D. 2006. Testing the Cenozoic multisite composite $\delta 180$ and $\delta 13 \mathrm{C}$ curves: new monospecific Eocene records from a single locality, Demerara Rise (Ocean Drilling Program Leg 207). Palaeoceanography, 21:PA2019. doi: 10.1029/2005PA001253

Siedler, G.; Gould, W. J. \& Church, J. 2001. Ocean Circulation and Climate: Observing and Modelling the Global Ocean. San Diego, Academic Press, 715 p. (International Geophysics Series 77).

Sluijs, A.; Brinkhuis, H.; Stickley, C.E.; Warnaar, J.; Williams, G.L. \& Fuller, M. 2003. Dinoflagellate cysts from the Eocene / Oligocene transition in the Southern Ocean; results from ODP Leg 189. In: N.F. Exon; J.P. Kennett \& M.J. Malone (eds.) Proceedings of the Ocean Drilling Program, College Station, Ocean Drilling Program, p. 1-42. (Scientific Results 189). doi:10.2973/odp.proc.sr.189.104.2003

Sluijs, A.; Brinkhuis, H.; Williams, G.L. \& Fensome, R.A. 2009. Taxonomic revision of some Cretaceous-Cenozoic spiny organic-walled peridiniacean dinoflagellate cysts. Review of Palaeobotany and Palynology, 154:34-53. doi:10.1016/j. revpalbo.2008.11.006

Sluijs, A.; Schouten, S.; Pagani, M.; Woltering, M.; Brinkhuis, H.; Sinninghe Damsté, J.S.; Dickens, G.R.; Huber, M.; Reichart, G.; Stein, R.; Matthiessen, J.; Lourens, L.J.; Pedentchouk, N.; Backman, J.; Moran, K., \& scientists, E. 3. 2006. Subtropical Arctic Ocean temperatures during the Palaeocene/Eocene thermal maximum. Nature, 441:610-613. doi:10.1038/ nature 04668

Sluijs, A.; Pross, J. \& Brinkhuis, H. 2005. From greenhouse to icehouse; organic-walled dinoflagellate cysts as paleoenvironmental indicators in the Paleogene. Earth Science Reviews, 68:281-315. doi:10.1016/j.earscirev.2004.06.001

Smayda, T.J. 2002. Turbulence, watermass stratification and harmful algal blooms: an alternative view and frontal zones as 'pelagic seed banks'. Harmful Algae, 1:95-112. doi:10.1016/S15689883(02)00010-0

Soto, M.; Morales, E.; Veroslavsky, G.; De Santa Ana, H.; Ucha, N. \& Rodríguez, P. 2011. The continental margin of Uruguay: crustal architecture and segmentation. Marine and Petroleum Geology, 28:1676-1689. doi:10.1016/j.marpetgeo.2011.07.001 
Stickley, C.E.; Brinkhuis, H.; Schellenberg, S.A.; Sluijs, A.; Röhl, U.; Fuller, M.; Grauert, M.; Huber, M.; Warnaar, J. \& Williams, G.L. 2004a. Timing and nature of the deepening of the Tasmanian Gateway. Paleoceanography, 19:PA4027. doi:10.1029/2004PA001022

Stickley, C.E.; Brinkhuis, H.; McGonigal, K.L.; Chaproniere, G.C.H.; Fuller, M., Kelly, D.C.; Nürnberg, D.; Pfuhl, H.A.; Schellenberg, S.A.; Schoenfeld, J.; Suzuki, N.; Touchard, Y.; Wei, W.; Williams, G.L.; Lara, J. \& Stant, S.A. 2004b. Late Cretaceous-Quaternary biomagnetostratigraphy of ODP Sites 1168, 1170, 1171, and 1172, Tasmanian Gateway. In: N.F. Exon; J.P. Kennett \& M.J. Malone (eds.) Proceedings of the Ocean Drilling Program, Scientific Results, 189:1-57. doi:10.2973/ odp.proc.sr.189.111.2004

Stoakes, F.A.; Campbell, C.V.; Cass, R. \& Ucha, N. 1991. Seismic stratigraphic analysis of the Punta del Este Basin, offshore Uruguay, South America. American Association of Petroleum Geologists Bulletin, 75:219-240.

Stover, L.E. 1975. Observations on some Australian Eocene dinoflagellates. Geoscience and Man, 11:35-45.

Stover, L.E. \& Evitt, W.R. 1978. Analyses of pre-Pleistocene organic-walled dinoflagellates. Stanford, Stanford University Publications, $300 \mathrm{p}$.

Stover, L.E. \& Williams, G.L. 1995. A revision of the Paleogene dinoflagellate genera Areosphaeridium Eaton 1971 and Eatonicysta Stover and Evitt 1978. Micropaleontology, 41:97141. doi:10.2307/1485947

Tavella, G.F. \& Wright, C.G. 1996. Cuenca del Salado. In: V.A. Ramos \& M.A. Turic (eds.) Geología y Recursos Naturales de la Plataforma Continental Argentina, Relatorio del $13^{\circ}$ Congreso Geológico Argentino y $3^{\circ}$ Congreso de Exploración de Hidrocarburos, Association Geologica Argentina, p. 95-116.

Truswell, E.M. 1997. Palynomorph assemblages from marine Eocene sediments on the West Tasmanian continental margin and the South Tasman Rise. Australian Journal of Earth Sciences, 4:633-654.

Ucha, N.; de Santa Ana, H. \& Veroslavsky, G. 2003. La cuenca Punta del Este: geología y potencial hidrocarburífero. In: G. Veroslavsky; M. Ubilla \& S. Martínez (eds.) Cuencas Sedimentarias de Uruguay: Geología, Paleontología y Recursos Naturales, Facultad de Ciencias, Universidad de La República, p. 171-190.

Uchupi, E. \& Emery, K. 1991. Pangaean divergent margins: historical perspective. Marine Geology, 102:1-28. doi:10.1016/00253227(91)90003-M

Urien, C.M. \& Zambrano, J.J. 1996. Estructura de la Plataforma Continental Argentina. In: V.A. Ramos \& M.A. Turic (eds.) Geología y Recursos Naturales de la Plataforma Continental
Argentina, Relatorio del $13^{\circ}$ Congreso Geológico Argentino y $3^{\circ}$ Congreso de Exploración de Hidrocarburos, Association Geologica Argentina, p. 29-66.

Vandenberghe, N.; Speijer, R.P. \& Hilgen, F.J. 2012. The Paleogene period. In: F.M. Gradstein; J.G. Ogg; M. Schmitz \& G. Ogg (eds.) The Geologic Time Scale 2012: Elsevier, p. 855-922. doi:10.1016/B978-0-444-59425-9.01001-5

Wall, D. 1967. Fossil microplankton in deep-sea cores from the Caribbean Sea. Palaeontology, 10:95-123.

Williams, G.L.; Brinkhuis, H.; Pearce, M.A.; Fensome, R.A. \& Weegink, J.W. 2004. Southern Ocean and global dinoflagellate cyst events compared: Index events for the late Cretaceous Neogene. In: N. Exon \& J.P. Kennett (eds.) Proceedings of the Ocean Drilling Program, College Station, Ocean Drilling Program, p. 1-98. (Scientific Results 189). doi:10.2973/odp. proc.sr.189.107.2004

Williams G. L.; Damassa, S.P.; Fensome, R.A. \& Guerstein, G.R. 2015. Wetzeliella and its allies - the 'hole' story: a taxonomic revision of the Paleogene dinoflagellate subfamily Wetzelielloideae, Palynology, 39:289-344. doi:10.1080/01916 122.2014.993888

Williams, G.L. \& Downie, C. 1966. Further dinoflagellate cysts from the London Clay. In: R.J. Davey; C. Downie; W.A.S. Sarjeant \& G.L. Williams (eds.) Studies on Mesozoic and Cainozoic dinoflagellate cysts, London, British Museum of Natural History, p.215-236. (Geology Bulletin Supplement 3).

Wilson, G.J. 1967. Some new species of Lower Tertiary dinoflagellates from McMurdo Sound, Antarctica. New Zealand Journal of Botany, 5:57-83. doi:10.1080/002882 $5 X .1967 .10428735$

Wrenn, J.H. \& Beckman, S.W. 1982. Maceral, total organic carbon, and palynological analyses of Ross Ice Shelf Project site J9 cores. Science, 216:187-189. doi:10.1126/science.216.4542.187

Wrenn, J.H. \& Hart, G.F. 1988. Paleogene dinoflagellate cyst biostratigraphy of Seymour Island, Antarctica. Geological Society of America Memoirs, 169:321-448. doi:10.1130/ MEM169-p321

Zachos, J.C.; Dickens, G.R. \& Zeebe, R.E. 2008. An early Cenozoic perspective on greenhouse warming and carbon-cycle dynamics. Nature, 451:279-283. doi:10.1038/nature06588

Zachos, J.; Pagani, M.; Sloan, L.; Thomas, E. \& Billups, K. 2001. Trends, rhythms, and aberrations in global climate $65 \mathrm{Ma}$ to present. Science, 292:686-693. doi: 10.1126/science.1059412

Zachos, J.C.; Stott, L.D. \& Lohmann, K.C. 1994. Evolution of early Cenozoic marine temperatures. Paleoceanography, 9:353-387. doi:10.1029/93PA03266

Received in January, 2016; accepted in July, 2016. 
Apéndice 1. Otras especies mencionadas en el texto.

Appendix 1. Other species mentioned in the text.

Areosphaeridium diktyoplokum (Klumpp) Eaton, 1971

Deflandrea cygniformis Pöthe de Baldis, 1966

Cordosphaeridium fibrospinosum (Davey \& Williams) Davey, 1969 